Archives

\title{
Réception du modèle divisionnel en France au début des années soixante-dix à la lumière de l'étude des pratiques
}

Pierre-Antoine Dessaux et Jean-Philippe Mazaud

\section{(2) OpenEdition \\ 1 Journals}

Édition électronique

URL : http://journals.openedition.org/ccrh/1632

DOI : $10.4000 /$ ccrh. 1632

ISSN : $1760-7906$

\section{Éditeur}

Centre de recherches historiques - EHESS

\section{Édition imprimée}

Date de publication : 20 octobre 2000

ISSN : 0990-9141

\section{Référence électronique}

Pierre-Antoine Dessaux et Jean-Philippe Mazaud, « Réception du modèle divisionnel en France au début des années soixante-dix à la lumière de l'étude des pratiques », Les Cahiers du Centre de Recherches Historiques [En ligne], 25 | 2000, mis en ligne le 16 janvier 2009, consulté le 20 avril 2019. URL : http://journals.openedition.org/ccrh/1632 ; DOI : 10.4000/ccrh.1632

Ce document a été généré automatiquement le 20 avril 2019.

Article L.111-1 du Code de la propriété intellectuelle. 


\title{
Réception du modèle divisionnel en France au début des années soixante-dix à la lumière de l'étude des pratiques
}

\author{
Pierre-Antoine Dessaux et Jean-Philippe Mazaud
}

\section{NOTE DE L'AUTEUR}

Le présent article est fondé sur des travaux encore en cours dans le cadre de doctorats ; il doit être considéré comme un propos d'étape qui fera l'objet de remaniements ultérieurs.

\section{Introduction}

1 Du milieu des années soixante à la crise de 1974, l'industrie française a connu un important mouvement de concentration s'appuyant sur des fusions qui furent l'occasion d'une transformation des pratiques de gestion que les courants "modernisateurs » appelaient de leurs vœux depuis le début de la décennie ${ }^{1}$. Dans un contexte de préparation des entreprises françaises à l'intensification supposée de la concurrence avec le renforcement du Marché commun et de reconnaissance de la prédominance des entreprises multinationales d'origine américaine qui tendaient alors à renforcer leur présence en Europe, le débat industriel s'orienta largement vers l'analyse des performances relatives des entreprises françaises et de leurs causes ${ }^{2}$. Une large part des analyses se focalisa sur le facteur taille, que ce dernier soit expliqué par un prétendu «malthusianisme» des entrepreneurs français ou, de façon moins polémique, par les limites du marché intérieur, les caractéristiques institutionnelles et sociales de la pratique des affaires dans ce cadre national, ou encore par les limites de la professionnalisation des dirigeants d'entreprises à la fois causes et conséquences de 
l'émiettement du tissu industriel. La «solution » aux problèmes de performance relative de l'industrie française fut donc identifiée, bien avant le début de la décennie, dans la croissance de la taille des entreprises soit provoquée par des incitations aux regroupements au sein de secteurs d'activité, soit résultant de la diversification de leurs activités, et censées conduire à une rationalisation des structures et des méthodes de gestion industrielle. De la croissance des entreprises devait en effet résulter la "modernisation" de leur mode de fonctionnement, la professionnalisation de leurs équipes, la rationalisation de leur structure dans la voie ouverte par les grandes entreprises américaines et permettant en conséquence d'envisager de pouvoir faire face à leur concurrence. Une large part de ces réorganisations se fondit en conséquence sur les modèles d'organisation mis en place par les managers des entreprises américaines et recourut à une utilisation remarquable des cabinets de consultants américains ${ }^{3}$.

2 Le paradigme alors dominant de l'analyse du développement des puissances industrielles guidait une large part de ces démarches de «modernisation $»^{4}$. La grande entreprise multidivisionnelle, fondée sur une hiérarchie salariée, emblématique de la puissance du capitalisme américain, traçait l'horizon naturel des dynamiques de nombreux secteurs d'activité. La capacité de domination des règles du jeu concurrentiel associée au développement précoce de telles formes aux États-Unis semblait indiquer la nature des moyens sur la base desquels devait se fonder la compétition entre ces institutions. L'évolution prenait en conséquence un caractère «naturel » et nécessaire. Une large part de l'historiographie relative à ces questions se développa autour de ce paradigme tout en contribuant à le renforcer. Autour de la notion de modèle unitaire de développement des organisations industrielles se multiplièrent les débats sur les étapes de son déploiement, sur les obstacles rencontrés en fonction des contextes nationaux, sur la pertinence d'hypothèses de particularismes institutionnels ou sociaux pouvant contribuer à les expliquer. En dépit de la reconnaissance précoce de l'existence de "modèles» différenciés d'entreprises capitalistes ${ }^{5}$, se maintint l'idée d'une évolution homogène conduisant à les percevoir d'abord comme des trajectoires que l'approfondissement de la concurrence conduirait à laminer 6 . Puisque la messe était dite, les démarches de réorganisation conformes à l'évolution attendue pouvaient être interprétées comme une «modernisation » assurant l'acquisition des capacités nécessaires au renforcement de la compétitivité des entreprises concernées. Les éventuelles réticences qu'elles pouvaient rencontrer relevaient alors de "résistances au changement » liées à des tensions internes à ces institutions ou à des particularismes venant renforcer l'hypothèse des modèles nationaux. Dans les deux cas, une "réforme» adéquate, de plus ou moins grande ampleur, pouvait permettre d'envisager une normalisation conforme à la dynamique générale. L'appréhension du modèle d'organisation, sa réception comme son éventuelle adaptation, sa mise en pratiques, portaient d'autant moins à débats que la logique d'évolution se voulait unitaire.

3 Une historiographie complémentaire voire concurrente s'est développée sur ces questions à partir des années quatre-vingts ${ }^{7}$. Discutant ce modèle de développement du capitalisme, elle s'est penchée de façon moins formelle sur les conditions de fonctionnement des structures industrielles, sur les pratiques qui furent au fondement de modes d'exercice de l'activité industrielle pour ouvrir des perspectives relatives à leur possible diversité et à l'approfondissement de l'analyse des configurations dans lesquelles elles se situaient. Ainsi, au sein même du modèle de référence, une attention nouvelle a été portée aux conditions sociales de mise en place de la grande entreprise. Olivier Zunz 
a, par exemple, proposé de lever le voile sur la constitution du groupe des cadres salariés des grands établissements du début du $\mathrm{xx}^{\mathrm{e}}$ siècle ${ }^{8}$. Dans la lignée de Michael Burawoy, Robert Freeland a tenté une relecture de la mise en place de la forme en $\mathrm{M}$ au sein du cas de référence qu'est General Motors pour souligner l'importance des considérations de gestion de l'adhésion des cadres relativement à la seule mise en forme organisationnelle de l'activités. Par ailleurs l'idée d'une multiplicité possible des configurations industrielles sous-jacentes à la mise en place d'un système de production de masse s'est, avec le développement du débat sur le modèle japonais, progressivement consolidée ${ }^{10}$. Les renouvellements de l'histoire sociale des techniques ont enfin considérablement contribué à amender l'idée d'un déterminisme structurel et souligné la complexité des trajectoires des organisations y puisant leur dynamique. L'ensemble de ces travaux invite à sortir des observations souvent très générales que l'on porte sur cette conjoncture pour mieux tenir compte des pratiques, ambitions et réceptions, qui la forgèrent et de leur potentielle diversité. Il s'agira alors de relire cet épisode pour en rechercher plus précisément les enjeux tant du point de vue des acteurs qui s'y impliquèrent, que de ceux qui furent alors mis en cause dans leurs façons de faire.

4 Un tel approfondissement se heurte évidemment à la question des sources qui lui sont nécessaires. L'absence de pratiques de versement, voire de conservation, des archives d'entreprises en France comparables à celles des sociétés américaines ${ }^{11}$, portées par un idéal d'exemplarité, rend l'exercice singulièrement difficile en particulier pour des périodes relativement récentes et pour les entreprises à caractère purement privé ${ }^{12}$. On s'appuiera donc ici sur un accès privilégié aux sources de deux entreprises extrêmement différentes, bien que concourant toutes deux à la production et à la diffusion en masse, la librairie Hachette, institution majeure du monde éditorial français, et la société Panzani ${ }^{13}$ acteur progressivement dominant d'un secteur secondaire de l'industrie alimentaire ${ }^{14}$. Ces deux entreprises firent pratiquement simultanément l'objet d'une réforme de leur mode d'organisation au tournant des années 1970. La librairie Hachette tenta, à l'occasion d'un changement d'équipe dirigeante, une formalisation de son activité dans le sens d'une organisation multi-divisionnelle. La société Panzani fut, quant à elle, intégrée au groupe BSN en 1973, transformée en filiale et soumise à la logique divisionnelle alors mise en place par le groupe. Dans les deux cas, le recours à des sources externes avec une focalisation sur l'évolution de l'organisation de ces sociétés et de leurs stratégies déclarées conduirait aisément à conclure à une " modernisation » par l'amélioration des principes de gestion, le développement de stratégies de diversification qui perdurèrent, la transformation $\mathrm{du}$ groupe dirigeant et surtout la consolidation d'une position hégémonique dans leurs domaines d'activité respectifs. On se propose donc de revenir ici sur cette évolution et le sens de la qualification qui peut lui être attribuée. En retraçant en premier lieu les pratiques qui étaient au cœur de l'activité de ces sociétés, il s'agira ensuite de tenter de saisir les motivations du changement des années soixante-dix et la profondeur de son impact. En effet, dans ces deux cas se retrouve une dynamique apparemment essentiellement motivée par des facteurs externes à l'activité courante qui conduisent à s'interroger sur les conditions de sa conception comme de sa mise en œuvre. Cette configuration étant relativement générale dans un contexte de grande faveur d'un modèle jugé, au double sens du terme, performant, elle pourrait permettre de questionner le sens que l'on pourrait prêter à cet épisode de « modernisation ». 


\section{Deux métiers orientés vers la diffusion de masse}

$5 \mathrm{Au}$ jugement «d'archaïsme » couramment porté, depuis les années 1960, sur les structures économiques françaises, l'étude des entreprises engagées dans la construction d'activités orientées vers la diffusion de produits de masse permet d'apporter de sérieuses réserves. En effet, par delà les effets de taille et de structure, les traces de la pratique des entrepreneurs engagés dans cette croissance permettent d'observer un remodelage continu de leurs activités comme de leurs produits pour construire des positions originales fondées sur de véritables savoir-faire. Ainsi, l'extension de la diffusion du livre sous l'impulsion de la librairie Hachette, entreprise plus que centenaire et toujours gérée par des descendants de son fondateur Louis Hachette, témoigne d'un travail en profondeur des circuits de distribution traditionnels de la librairie. Ce développement permet également de saisir les facultés d'adaptation d'une société dominante dans son secteur à la variété du monde de la librairie et de l'édition. Comparable par la progression de ses ventes, la société Panzani offre le point de vue complémentaire d'un entrepreneur parti de rien et engagé dans la construction d'un position dominante dans le secteur des pâtes alimentaires par un effort constant d'adaptation aux grands distributeurs, effort étonnamment symétrique de celui de la librairie. Ces deux métiers d'artisans, dans leurs domaines respectifs, de la croissance d'après-guerre, peuvent, en première analyse, être abordés à partir des pratiques professionnelles de leurs dirigeants.

\section{Le métier original de la direction de la librairie Hachette}

\section{La construction de la librairie Hachette :}

\section{Le travail de massification des ventes de livres}

6 L'analyse du métier de la librairie Hachette nous impose un détour par l'histoire de sa construction qui se caractérise par un aller-retour incessant entre l'édition et la distribution des livres. Dès les débuts de son entreprise en 1826, Louis Hachette ne se contente pas de sa fonction d'éditeur de livres scolaire et de littérature enfantine: il cherche très vite à atteindre de nouvelles clientèles en installant des circuits de distribution inconnus en France grâce au réseau des bibliothèques de gare. Par un travail continu d'articulation entre vente et création éditoriale, poursuivi par les héritiers du fondateur, le produit livre incorpore le moyen de sa distribution dans un remodelage constant : ainsi de nombreux ouvrages sont-ils édités pour être vendus dans la collection bien nommée de la «Bibliothèque des chemins de fer ». Les «Guides touristiques »" la «Bibliothèque rose ", et plus tard la "Bibliothèque verte » deviennent rapidement les produits phares de la librairie Hachette dont le succès repose d'abord sur leur mode de distribution.

$7 \mathrm{Au}$ cours du $\mathrm{xx}^{\mathrm{e}}$ siècle, la librairie Hachette ne cesse d'approfondir cette définition conjointe du livre et du service de distribution. À partir de 1897, l'acquisition de sociétés de messagerie sur tout le territoire lui facilite l'accès aux lieux de consommation urbains. Mais la librairie Hachette n'omet pas d'intégrer ces services dans une nouvelle extension $\mathrm{du}$ produit livre. Elle s'efforce ainsi de prendre en charge la distribution de romans populaires édités dans des formes très similaires à celles de la presse. Les romans populaires sentimentaux ou policiers connaissent un fort succès grâce à leur vente en 
collection par les marchands de journaux ${ }^{16}$. Informée du succès de ces nouveaux circuits ${ }^{17}$ , la librairie Hachette procède à l'acquisition de la maison d'édition de P. Lafitte en 1916 et prend des participations chez Tallandier, éditeur du Livre nationa ${ }^{18}$.

8 Dans les années 1930, les recettes de ce savoir-faire commercial sont à nouveau reprises et approfondies : les succès obtenus dans les circuits populaires permettent de songer à étendre les modalités de distribution de la « petite librairie » à la « librairie générale ». En effet, la maîtrise de ces circuits confère à la librairie Hachette une capacité d'évaluation de la probabilité de vente des ouvrages dans les lieux de la consommation quotidienne (kiosques, petits dépositaires) qui séduit les éditeurs de littérature. En 1932, Gallimard signe un contrat donnant l'exclusivité des ventes de son fonds à la librairie Hachette. Ce contrat scelle une coopération étroite tout en conservant l'autonomie des deux maisons. Gallimard réduit son risque de mévente grâce aux conditions particulières accordées par le distributeur, et la librairie Hachette obtient les moyens d'alimenter ses circuits de vente en puisant dans le fonds éditorial d'une maison qu'elle n'a pas à gérer. La librairie Hachette gagne le droit de rééditer un pourcentage variable d'auteurs à succès choisis dans le fonds Gallimard. En échange, elle garantit à l'éditeur la prise en compte ferme de $75 \%$ de ses tirages. Pour sa propre rémunération, Hachette accepte une moindre remise de l'éditeur, anticipant la croissance des ventes attendue de ce partenariat. Ainsi, l'interpénétration des deux entreprises se caractérise par un transfert des risques de l'éditeur sur le distributeur, tandis que le risque du distributeur est lui-même amoindri par la garantie d'approvisionnement que lui offre l'accès à un fonds éditorial sans égal. La même dynamique se poursuit en 1938, lorsque la librairie Hachette fixe dans un contrat passé entre sa filiale, la Librairie Générale Française, et Calmann-Lévy les principes de la "collection pourpre" où seront réédités les grands auteurs de cette maison dans un format bon marché. En partageant les investissements et les profits issus de cette collection avec Calmann-Lévy, la librairie Hachette augmente non seulement la possibilité de rééditer des ouvrages à grand succès, mais acquiert aussi le droit de les présenter sous une collection nouvelle destinée à la vente dans ses circuits.

La spécificité de ce commerce se laisse entrevoir en priorité dans les relations continues des dirigeants de la librairie avec le monde de l'édition dont ils sont, à la fois, un pilier commercial et financier ${ }^{19}$. Comprendre la croissance de la diffusion du livre par le réseau de distribution de la librairie Hachette impose de considérer la multiplication des contrats passés entre les éditeurs soucieux de se donner un accès relativement sûr à la clientèle et les dirigeants de l'appareil de distribution de la librairie. Ces contrats mettent en jeu des relations hybrides entre sous-traitance et partenariat entre pairs qui fonctionnent dans un système relationnel particulier et dont la gestion, pour l'aprèsguerre, mérite d'être explicitée.

\section{Des comptes rendus de réunions hebdomadaires à trois}

10 Les documents déposés dans les sous-sols du siège de Hachette nous donnent les moyens de percevoir la façon de diriger qui a permis d'accroître le volume des ventes de livres ${ }^{20}$. De 1947 à 1967, l'essentiel du corpus de sources écrites sur l'activité de dirigeant, dans la librairie Hachette, consiste en comptes rendus, dactylographiés mais relativement peu rédigés, des réunions hebdomadaires entre Robert Meunier-adjoint et cousin du président Edmond Fouret à qui il succède en 1952 - Henri Gautrelet, son bras droit, et Henri Filipacchi, le responsable des services commerciaux ${ }^{21}$. Deux de ces personnages sont peu à peu rejoints, puis remplacés par le neveu de Robert Meunier, Ithier de 
Roquemaurel, et Guy Schoeller prenant la suite d'Henri Filipacchi après sa disparition en septembre $1961^{22}$. Y sont prises les décisions de financement et de commercialisation d'ouvrages, essentiellement des petites décisions, qui orientent les étapes de la négociation avec chaque éditeur, les mesures d'économie à prendre, etc. ${ }^{23}$. Leur dépouillement permet d'observer que ce sont toujours les mêmes questions qui reviennent et auxquelles répond, après une discussion qui n'est pas retranscrite, un arbitrage prononcé par Robert Meunier. Ce corpus apparaît très homogène dans la mesure où il fait intervenir toujours les mêmes personnes, où il traite toujours des mêmes questions. Ce sont ainsi des informations partielles mais continuellement reprises qui forment l'essentiel de la trace des négociations; elles permettent de comprendre comment la librairie Hachette construit ses positions, et l'exemple suivant, tiré de la réunion du 9 avril 1947, peut en éclairer le fonctionnement ${ }^{24}$ :

- Javel : M. Filipacchi a pu réduire son personnel de 58 personnes $(550000)$ en ne remplaçant pas les départs.

- Règlements : M. Ferenczi refuse le règlement par traite à 90 jours. M. Meunier le verra.

- La Jeune Parque : son compte apparaît débiteur de $1200000 \mathrm{~F}$ par suite de gros retours en février et mars.

11 Ces notes sommaires, régulièrement rédigées par Henri Gautrelet, qui assume la tâche de suivre l'exécution des décisions, forment des séries d'arrêts sur les positions de Hachette dans ses négociations avec les éditeurs, mais aussi avec la partie commerciale qui anticipe la demande. Elles témoignent également d'une discussion permanente entre le représentant de la partie commerciale et le président de Hachette qui, par les avances financières qu'il concède sur les stocks d'éditeurs, permet à ces derniers de trouver une garantie relative à la vente de leurs livres. Elles s'articulent entre les clientèles auxquelles les circuits de vente donnent accès et les éditeurs que Robert Meunier soutient en rendant possible la production des publications dont les ventes sont alors pratiquement acquises. Ces discussions révèlent aussi une préoccupation permanente portant sur la trésorerie. En effet, Hachette souhaite imposer les conditions de paiement qui lui seront le plus favorables. Les comptes rendus de réunion suggèrent que l'activité de supervision du président directeur général se résume à la tâche de coordonner, grâce à des moyens financiers importants, les offres des éditeurs à un réseau de distribution stabilisé à la fin du XIX ${ }^{e}$ siècle $^{25}$. Outre ce regard d'exploitant grossiste, plusieurs enseignements peuvent être tirés des relations particulières qui se nouent avec les éditeurs périphériques et la librairie.

12 En premier lieu, le nombre de partenaires de la librairie Hachette est important: les comptes rendus les moins riches évoquent un ou deux éditeurs, mais certains concernent une demi-douzaine de négociations courantes. Chaque réunion examine un sujet ou deux relativement à chaque partenaire. Tout partenaire est dans un rapport particulier avec la librairie, et ce rapport est géré au cas par cas. Ainsi voit-on le chef de la famille Hachette mettre toute son autorité dans la négociation des conditions de trésorerie qu'il demande auprès d'un autre entrepreneur du livre en la personne de l'éditeur de littérature populaire Ferenczi. En revanche, la Jeune Parque, jeune maison novatrice mais fragile est surveillée de près, et l'on sent le moment où Hachette va être sollicitée pour lui venir en aide.

13 De plus, les sujets abordés mettent en débat des questions d'argent projet par projet. Il ne s'agit pas de chiffres trop lourds, mais de petites conditions de crédits, de remises. Les noms des éditeurs reviennent périodiquement pour évoquer l'état des rapports avec la 
librairie. Les négociations apparaissent comme une activité du quotidien. Les occasions de lancer des « nouveautés » émergent de cette pragmatique de la coopération au cas par cas avec les éditeurs. Les habitués du métier savent intimement que les projets radicalement neufs sont rares, mais ils sont attentifs aux modes de présentation des œuvres qui sont susceptibles de changer plus régulièrement. L'apparition du «Livre de poche » en 1953 n'est qu'un exemple particulièrement éclairant de la façon dont ces idées de " nouveauté » se concrétisent. La question du format bon marché est, depuis le XIX siècle, constamment remise sur le métier de la librairie Hachette, et le «Livre de poche » en est le dernier développement. Depuis l'initiative de la "Collection pourpre», Meunier observe les occasions de donner plus de portée à l'accord passé avec Calmann-Lévy : c'est lors de ces réunions hebdomadaires qu'il demande, dans une sorte de veille tactique, des informations techniques sur les couvertures souples. La réflexion sur la possibilité d'attirer les autres éditeurs sur une formule encore plus attrayante progresse ${ }^{26}$. Ainsi, aux petites améliorations de présentation de la "Collection pourpre » liées aux efforts du graphiste Maximilien Vox, succèdent celles attendues des nouveaux procédés de pelliculage qui permettent la fabrication de la couverture du «Livre de poche ». Sous des allures d'« innovation radicale », cette ultime formule de livre populaire s'est décidée lors d'une série de réunions dont le fonctionnement n'a pas été altéré pour ce projet. Son lancement en 1953 ne fait pas plus de bruit que les autres décisions courantes. En effet, la préparation du "Livre de poche » ne s'est pas coulée dans une procédure formelle, distincte des autres affaires du moment. À cet égard, il fut la continuation banale d'autres petits essais, d'autres réussites de collections populaires.

Les grandes affaires de la librairie sont la résultante de l'approfondissement de ce métier de négociations. Nul besoin donc de formalisation. Au contraire, les traces écrites de ces négociations laissent entrevoir l'importance des relations informelles, orales. Tout n'est pas relevé des interactions entre les participants des réunions qui fonctionnent selon des implicites communs. L'évaluation de l'état des «retours", catégorie élémentaire du métier d'éditeur qui permet de se rendre compte de ce qui se vend bien, y est ainsi systématique. La connaissance des tendances est donc partagée, mais les motifs éditoriaux des choix ne sont pas formellement discutés dans les comptes rendus. Comment expliquer par exemple le soutien à la Jeune Parque et au Chêne? Le corpus trouve là des limites que l'on peut combler par la connaissance, alors diffuse, des succès d'Henry Miller publiés par le Chêne, dont la censure s'entête à interdire les ouvrages.

Le fonctionnement de ces réunions, à l'origine de la vente en masse, se perçoit à travers la réitération d'échanges d'informations partielles, que mentionnent les comptes rendus, sur le mode implicite des connaissances partagées. Il se caractérise par des interactions se déroulant dans l'entre-soi du monde des entrepreneurs de livres, construit au gré des négociations entre les responsables et les éditeurs partenaires de la librairie Hachette. La continuité de ces discussions, la volonté des acteurs du métier de s'ajuster pour maximiser les ventes en fonction des succès anticipés et des retours des circuits de distribution, expliquent l'inutilité d'une formalisation des décisions, qui ne sont que des compromis momentanés. Par ailleurs, les notes prises au cours de ces réunions chez Meunier entérinent les positions arrêtées par l'exercice d'un pouvoir rarement mis en cause : celui d'un chef d'entreprise disposant de l'autonomie la plus large pour financer une multitude de petits projets. Aussi cette autorité n'éprouve-t-elle pas le besoin de justifier la façon dont la direction générale est elle-même organisée. L'ensemble de ces 
pratiques est en effet fondé sur une capacité financière qui permet à Robert Meunier, dont

[...] la réputation est celle d'un homme sachant faire rentrer les profits ${ }^{27}$, de n'avoir de comptes à rendre à personne, et ainsi d'échapper à une normalisation de ses méthodes de travail personnelles.

\section{Un mode de fonctionnement fondé sur une domination financière du métier, à l'écart des problèmes d'organisation interne}

16 A quoi tient ce fonctionnement? Les comptes rendus des réunions "chez Meunier " montrent assez clairement que l'essentiel de l'activité tient d'une part à la capacité financière du président de la librairie Hachette, et d'autre part à un choix des projets à promouvoir qui se fonde sur le retour des informations des services commerciaux du livre.

17 La capacité financière de Robert Meunier repose sur l'indépendance que lui accorde la Banque de Paris et des Pays-Bas, premier actionnaire bancaire de la société Hachette, et sur les positions de la librairie dans le commerce de détail. Disposant d'une capacité discrétionnaire d'orientation de la trésorerie, Meunier tient une clientèle d'éditeurs. Ces derniers remettent une partie de leur destin commercial et financier entre ses mains. Lorsque pour des raisons de prestige ou des mouvements d'humeur, il s'est fait trop interventionniste, les hommes de l'art, avec qui il entretient des relations de «chef de maison » à "chef de maison", n'ont cependant pas manqué de lui résister. Étant luimême du métier, il en comprend et accepte les règles ${ }^{28}$.

18 La réunion hebdomadaire avec Henri Filipacchi, responsable de la partie commerciale, et Henri Gautrelet, l'ingénieur centralien considéré comme l'homme des chiffres, vise à évaluer les projets éditoriaux porteurs dans lesquels la librairie peut s'engager en avançant le prix des stocks de livres aux éditeurs en fixant les clauses de reprises souhaitables pour la librairie. Elle évite ainsi de supporter elle-même le risque de mévente. De plus dans une économie de la variété, où le succès d'un titre permet de récupérer la mise sur plusieurs échecs, des expériences peuvent être tentées pour créer la nouveauté. Cela suppose de supporter au jour le jour la vie des petits éditeurs qui se sont positionnés sur un créneau, ou jouent des opportunités qui se présentent. Pour peser et soupeser les risques, les contacts informels avec les connaisseurs comme Henri Filipacchi sont indispensables.

Les traces de la supervision de Robert Meunier sur l'organisation interne sont beaucoup plus rares: cette absence révèle que l'organisation est considérée comme une affaire annexe. Cette position le maintient dans le monde des éditeurs, dans lequel la famille Hachette a toujours souhaité inscrire son travail : c'est comme éditeur, voire comme coéditeur que Robert Meunier traite avec Calmann-Lévy, ou Gallimard. L'image de l'industriel soucieux de rentabiliser des coûts d'organisation importants indisposerait sa clientèle ${ }^{29}$. Dans ses relations avec les éditeurs, la librairie Hachette, en sélectionnant les livres à diffuser, construit une solution à la contradiction socialement construite entre l'œuvre littéraire et la marchandise. La solution d'un tel paradoxe est fragile: elle ne saurait reposer sur la prééminence de l'aspect industriel sur l'aspect créatif, et chaque éditeur reste jaloux de sa réputation. Aussi les dirigeants de la librairie Hachette ne souhaitent-ils en rien valoriser les profits qu'ils tirent d'une rationalisation des efforts de distribution et de présentation des livres. Le «Livre de poche», vecteur de la 
massification des ventes, est une appellation d'allure générique où le nom de la librairie Hachette n'apparaît pas. De plus, c'est une société filiale, la Librairie Générale Française, qui le publie. L'éditeur qui a découvert l'œuvre figure parmi les mentions obligatoires imprimées dans les premières pages des livres de la collection. Dans ces conditions l'intermédiation de Hachette entre les éditeurs et les clientèles est présentée, et probablement vécue, comme un service rendu à la profession, où la question de l'amortissement des coûts de structure n'est que secondaire par rapport à l'objectif d'une diffusion sans cesse élargie.

Si le souci de planifier, d'organiser l'activité ne trouve guère de place dans les dossiers du président Meunier, c'est qu'en réalité la rationalisation des tâches matérielles a, déjà, bien eu lieu. La discrétion d'usage sur ce sujet montre que la distinction entre tâches éditoriales, d'essence artisanale, et tâches de distribution "scientifiquement organisées " sert l'image d'une librairie Hachette dont les racines sont les mêmes que toutes les autres maisons d'édition du quartier latin. Robert Meunier travaille dans les locaux historiques de la librairie. C'est au boulevard Saint-Germain que les dirigeants familiaux et leurs «collaborateurs» font leur apprentissage. Là, le coup d'œil du chef de famille est permanent. Cette atmosphère des locaux du «Boulevard» diffère de celle du centre de "Javel », où se trouvent les dépôts et les services commerciaux du livre. Dans ce dernier établissement, les employés sont assignés à des tâches très organisées. L'« artisanat éditorial » est réservé aux gens du siège, tandis qu'une organisation militarisée, soutenue par une hiérarchie de cadres, du brigadier au directeur, encadre le travail employé des locaux de "Javel». Un document de 1942 décrit formellement «le contrôle Hachette » présenté comme un véritable système, graphiques à l'appui ${ }^{30}$. Ces pratiques de travail dont les normes sont pensées depuis la mise en place des messageries Hachette après la Première Guerre mondiale, contrastent avec celles des départements d'édition, sis au siège de la librairie.

21 Ainsi, les positions sociales, mais aussi marchandes, gagnées par les membres de la famille Hachette et leurs cadres maisons, font d'eux des intermédiaires capables de résoudre la tension qui existe entre un monde des éditeurs, marqué par la reconnaissance des succès littéraires, biens symboliques se voulant rare, et un monde commercial, dont les règles de fonctionnement tendent à rationaliser un effort de captation des clientèles sur tous les lieux possibles de consommation de l'imprimé. Cela permet aux éditeurs comme aux dirigeants de la librairie Hachette d'assumer ensemble des positions créatives, tout en laissant aux services commerciaux le soin d'une organisation besogneuse et efficace des circuits de distribution qui font du livre et des grands auteurs des produits du quotidien. C'est dans le cadre de ce métier hybride, entre production et distribution, dont les offres intègrent les aspirations de clientèles variées, que se réalise la massification de la diffusion des livres de « librairie générale » comme de « petite librairie $»^{31}$.

La puissance de la librairie Hachette, qui s'explique par les grandes disponibilités financières que lui procurent, à la fois, le marché financier et la trésorerie issue de la vente en aval des produits de l'édition, tient probablement également à cette structure de direction encore relativement souple, voire artisanale, parfaitement imprégnée des règles et de l'histoire d'un métier que ses décideurs ont contribué à façonner directement et qui lui permet une bonne réactivité. La modernisation du groupe qui, à la fin des années 1960, fait apparaître formellement les logiques industrielles de pôles d'activité distincts, le livre et la distribution, ne pouvait que remettre profondément en cause la permanence de cette forme de direction qui gérait le plus discrètement possible la relation complexe 
entre les œuvres des éditeurs et leur transformation en marchandise. Orientée vers des objectifs commerciaux, cette direction était sans doute plus particulièrement adaptée à un contexte d'anticipation favorable de la consommation.

\section{La transformation commerciale d'un métier industriel}

Si le cas de la librairie Hachette nous invite à observer un exemple de coordination sectorielle assurée par un acteur fortement impliqué dans la distribution d'un produit extrêmement diversifié, celui de la société Panzani peut être conçu, en dépit de la distance entre les domaines d'activité, comme relativement complémentaire. Du point de vue du distributeur il nous permet de passer à celui du producteur, de la variété des produits à celui d'une apparente homogénéité tout en demeurant dans le cadre de la mise en forme de moyens de diffusion en masse de produits manufacturés.

La croissance de la société Panzani, dont l'activité démarre durant les années quarante et devenue, au début des années soixante-dix dominante dans son secteur initial d'activité-les pâtes alimentaires-met en question les modèles traditionnels de croissance des entreprises essentiellement fondés sur l'exploitation des moyens internes ${ }^{32}$. Fondée tardivement dans un marché déjà très encombré33, elle ne disposait d'aucune source discriminante de compétitivité. Les techniques de production ne pouvaient laisser entrevoir de potentiel de gains de productivité majeurs, le produit était faiblement différentiable et la société, initialement personnelle d'un entrepreneur sans fortune, Jean Panzani, ne pouvait envisager de recourir massivement aux investissements publicitaires et commerciaux qui auraient pu concourir à la construction d'une prédominance sur le marchés ${ }^{34}$. Pourtant, l'entreprise trouva les moyens d'une vive croissance : sa production doubla entre 1950 et 1958 de même que sa part de marché, ce dernier ne connaissant qu'une faible croissance. Elle entra alors dans une vague de fusions qui la portèrent, au début des années soixante-dix, au contrôle de plus de $40 \%$ de son marché. Ces fusions doublèrent à deux reprises la taille de la société ${ }^{35}$. Dans ces deux cas elles résultaient de sociétés concurrentes, de dimension supérieure, qui trouvèrent là le moyen de donner un nouveau souffle à leur activité. Elles rachetaient ainsi non seulement un concurrent agressif mais encore une autre approche de l'activité, jugée plus dynamique, si l'on en croit la permanence de la direction opérationnelle du fondateur et de son équipe. C'est donc à ces derniers qu'il convient de se reporter pour saisir les motifs de cette capacité de concentration de ce secteur d'activité. Interrogé sur ce point, Jean Panzani ne fait nullement référence à des capacités de gestion particulières, elles furent en fait largement tirées de l'expérience des cadres issus des sociétés fusionnées ${ }^{36}$, ni à de sérieuses particularités du produit ou de la technique de production, mais résume ainsi, laconiquement, le fondement de sa démarche :

Je peux affirmer que j'ai inventé ou créé la relation directe, humaine, entre la

production et le négoce, avant de mettre en place la coopération. ${ }^{37}$

C'est dans la relation commerciale, de toute apparence peu soutenue par des particularités amont, qu'il conviendrait de rechercher la conception que cette entreprise se faisait de son métier et de sa valeur.

\section{Jouer sur les frontières de la relation client/fournisseur}

Ces pratiques commerciales peuvent être retracées à partir de la correspondance que la société échange avec sa clientèle. Parmi les courriers conservés dans les archives de la 
société, ceux relatifs au groupe Casino, dont elle devient un fournisseur à partir de la fusion de 1964, peuvent permettre de suivre comment se noue cette "relation directe». De février 1965 à juin 1966 ce sont ainsi près de 70 courriers, soit plus d'un par semaine, que Jean Panzani échange, directement bien qu'il dispose d'un service commercial de plus d'une centaine de salariés, avec Charles et Pierre Guichard qui dirigent alors ce grand groupe de distribution. C'est donc au plus haut niveau que se développe l'ensemble de la relation entre les deux sociétés depuis les négociations contractuelles jusqu'au suivi des problèmes épisodiquement posés par les livraisons. Ces courriers apparaissent doublés de visites réciproques qui permettent à Jean Panzani d'exposer les potentialités comme les limites de son appareil de production et de saisir le mode de fonctionnement de son nouveau client, son organisation, ses ambitions. En quelques mois, malgré des tensions, se construit ainsi une relation familière sur la base de laquelle il devient possible d'abord d'envisager plus sereinement les micro-conflits qui ne manquent pas de survenir entre le fournisseur et un client aussi important et surtout d'élaborer la mise en place de contrats spécifiques. Début 1966, les discussions aboutissent ainsi à une proposition de gestion directe de la concurrence interne aux rayons du distributeur. Fournisseur des marques de fabrique et de distribution, Jean Panzani propose la politique à suivre. Capable de croiser les résultats de son client avec ceux du reste de sa clientèle, il est en mesure de lui fournir une évaluation de sa position de marché :

Vos sorties de 1965 auront été très satisfaisantes, et se seront situées très largement au dessus de l'activité professionnelle et sensiblement au-dessus de celles du succursalisme en général, ${ }^{38}$

écrit-il à Charles Guichard. Enfin, sur un marché secondaire, il propose l'éviction de la concurrence en contrepartie d'une remise supplémentaire. Réciproquement, conscient des contraintes techniques, Charles Guichard lui concède une tolérance sur les erreurs de livraisons, lui transmet les informations nécessaires à l'amélioration de la gestion, l'informe parfois des conditions accordées par ses propres concurrents pour inviter son fournisseur à mettre de l'ordre dans sa clientèle.

On observe ainsi l'interpénétration de ces deux entreprises bien au-delà d'une relation contractuelle. L'articulation entre fournisseurs et distributeurs lie des entreprises dans une coopération conflictuelle. C'est sur la base d'une reconnaissance pragmatique de ce conflit d'intérêts inscrit dans un enjeu commun, une diffusion toujours plus massive, que Jean Panzani propose à ses interlocuteurs de placer leurs relations :

Votre fonction est d'acheter ; la mienne est de vendre, mais par delà les actes qui se doivent de rester positifs, il est incontestable que la collaboration productive doit prendre le pas sur le mieux-être de chacune des deux Sociétés que nous représentons. ${ }^{39}$

Il s'agit d'inscrire la relation dans un horizon temporel dépassant le cadre ponctuel du contrat et engageant en conséquence des investissements partagés contradictoires de la règle du profit immédiat pour construire par la «collaboration productive » d'autres opportunités d'affaires ${ }^{40}$. La diffusion en masse du produit passe ici par la mise en place d'une forme tout à fait singulière de " grands comptes ".

Cette volonté de "collaboration" vise, en modifiant les frontières des sociétés, à transformer sans parvenir à la supprimer la relation conflictuelle qui les lie. Ainsi Charles Guichard, actionnaire minoritaire de la nouvelle société issue de la fusion de 1964, cherche-t-il à obtenir des informations sur les coûts de production pour renforcer sa capacité de négociation en plaçant son fournisseur dans une relation dominée de soustraitance. Réciproquement Jean Panzani tire de l'information du mode de 
fonctionnement de son client des arguments visant à réduire ses prétentions dans la réalisation des contrats. Au-delà de cette accumulation continue de capacités de négociation, dont la marque et sa notoriété n'est qu'un élément, la proposition vise à établir une relation dans laquelle une claire définition des rôles et des objectifs communs permet une circulation de l'information privée qui structure un nouvel espace de pratiques. Ainsi Jean Panzani peut-il disposer d'une large part des données relatives à la gestion du produit interne à son client et se proposer comme prestataire de cette activité. Charles Guichard n'hésite d'ailleurs pas à en susciter l'amélioration en proposant à la discussion les pratiques en cours sur d'autres marchés, en particulier par les multinationales d'origine américaine qui conduisent, diffé-rentiellement, à préciser et donc à définir les spécificités du marché partagé ${ }^{41}$. Progressivement à la relation de pure négociation sur les termes de l'échange se substitue une réflexion conjointe sur le développement et la valorisation de l'activité adaptés au client. Derrière l'apparente banalité du produit émerge ainsi une dynamique de diversification des contrats qui fondent sa diffusion et instituent la société Panzani en prestataire d'écoulement du produit et de ses dérivés ${ }^{42}$.

Cet échantillon de correspondance éclaire le fondement de la croissance de l'entreprise et de sa marque. En attachant une attention particulière à la qualité des relations avec sa clientèle, Jean Panzani rechercha celle qui était le plus susceptible d'apprécier ce type d'approche et dont il put tirer les meilleurs résultats. Il se focalisa ainsi sur la portion de la distribution la plus concentrée et la plus structurée, susceptible de trouver des avantages à la sous-traitance d'une portion de son activité interne. Il plaça ainsi ses produits dans le segment dynamique de la transformation des réseaux de distribution après-guerre et bénéficia de sa croissance. Parallèlement il palliait les médiocres ressources de sa société par le recours aux systèmes d'information de sa clientèle et, le cas échéant, à leurs réseaux internes économisant ici en investissements de connaissance du marché et de constitution de réseaux commerciaux très ramifiés ${ }^{43}$. En contrepartie il acceptait donc des formes de négociation encore relativement éloignées des pratiques des industriels de l'alimentaire et en tira un réseau de relations qui fonda, en dépit de déboires capitalistiques ${ }^{44}$, le succès de son entreprise. En provoquant, dans son domaine, un développement de pratiques marchandes coopératives là où l'habitude était plutôt au placement ponctuel ou à la recherche des moyens d'exercice d'une domination unilatérale, il contribua à modifier son métier en l'ouvrant à la pratique des relations continues avec la distribution qui fonde la diffusion massive des produits banals. Cette capacité à redéfinir son secteur d'activité par le produit comme par les pratiques de diffusion explique probablement une large part de la dynamique de concentration d'entreprises importantes autour de sa société.

\section{Une organisation souple}

Les conséquences internes de cette pratique du métier peuvent être retracées à partir des documents laissés par les comités de direction que les actionnaires, initialement industriels indépendants, mettent en place à partir de 1964 pour contrôler l'activité de leur directeur général. Si les objectifs de la société sont clairs (assurer la rentabilisation d'une activité structurellement peu profitable du fait de la nature du produit et des contraintes de la politique de contrôle des prix qui pèsent sur les stratégies de valorisation), sa mise en œuvre apparaît difficile. Elle pâtit d'une concurrence qui commence à appliquer des méthodes similaires comme de la tendance de la clientèle à la 
favoriser pour éviter l'émergence d'un acteur trop dominant sur ce marché. D'autre part, la multiplication des contrats et des points de négociations ainsi que des relations à gérer renforce l'incertitude quant à la réalisation des prévisions. Directement engagé dans les relations commerciales, Jean Panzani tend à réagir très rapidement à la conjoncture de l'écoulement de ses produits et multiplie des tactiques soumises pour approbation à ses actionnaires. Des tensions se font bien sentir dans le sens d'une rationalisation de l'activité mais elles se heurtent à la réalité du métier. Ainsi trouve-t-on prégnant le leitmotiv de la « mise en ordre des relations commerciales » qui permettrait de simplifier la gestion des flux et de construire des prévisions plus solides. Mais l'échec d'une tentative d'unification des conditions commerciales développée en 1966 et 1967 sur la foi de la taille nouvellement acquise par la société conduit à écarter l'espoir d'une future simplification. La diversification est bien évoquée et mise en œuvre mais essentiellement pour rentabiliser les coûts commerciaux et assurer des revenus complémentaires car l'on sait l'effort que sa stabilisation impliquerait. En aucune façon elle ne fait l'objet d'investissements autres que commerciaux. La fuite en avant dans la rationalisation des structures est également mise en œuvre mais essentiellement dans le sens des économies internes car les incertitudes courantes conduisent à écarter un effort de productivité dont les résultats ne peuvent émerger qu'à terme. L'approche pratique que Jean Panzani a de son activité le conduit donc à concentrer son attention dans la réalisation courante du couple volume/prix, source majeure d'incertitude, plutôt qu'à concevoir d'autres perspectives pour son entreprise. En ce sens il fut bien l'homme de son métier, engagé dans sa transformation et non le gestionnaire d'une activité quelconque. Ce mode de fonctionnement plaçait assez logiquement au second plan les préoccupations relatives à l'organisation interne dont la formalisation eut supposé une plus grande stabilité des perspectives de l'entreprise. L'adaptation était bien plus nécessaire que l'optimisation des flux. Elle pouvait se fonder sur une large décentralisation et une évolution permanente des structure $\mathrm{s}^{45}$ ainsi que sur l'engagement d'un personnel intermédiaire très impliqué dans le fonctionnement de l'entreprise ${ }^{46}$. La pratique de négociation de contrats spécifiques à la clientèle trouvait ainsi les moyens de sa réalisation.

À l'heure de la diffusion en masse de leurs produits, les mondes du livre et des produits alimentaires se sont, en France, appuyés sur des réseaux de distribution relativement concentrés ${ }^{47}$. Cette caractéristique invite à envisager les interactions entre les acteurs d'entreprises distinctes mais complémentaires comme le lieu de pratiques de métiers apparemment plus importantes pour le développement des nouvelles échelles de la consommation qui se mettent en place durant les années soixante que les questions proprement structurelles dont l'importance fut beaucoup plus évidente dans d'autres secteurs en raison de la complexité des produits. Bien que parfaitement perçues par certains observateurs contemporains, ces différences furent mises à mal par la vogue du modèle gestionnaire américain au début des années soixante-dix. La rencontre entre ces univers de pratiques et le modèle divisionnel invite ainsi à observer la réception de ce dernier d'un point de vue peut-être un peu plus interne qu'il n'est d'usage de le faire. 


\title{
La réception du modèle d'organisation multidivisionnelle
}

\section{Chez Hachette}

\author{
Le regard de l'organisateur : \\ La préoccupation de mise en équivalence générale
}

31 Chez Hachette, les traces de la pratique dirigeante changent de forme après que Robert Meunier ait, en décembre 1967, cédé sa place à son neveu Ithier de Roquemaurel. Ce dernier ouvre son mandat par la commande d'une étude sur les structures de direction à la SEMA, une société de conseils, filiale de l'actionnaire Paribas. La "Société d'études et de mathématiques appliquées » créée à l'initiative de dirigeants de la banque au milieu des années 1960, proclame alors

[...] la nécessité pour les chefs d'entreprise de recourir à des disciplines modernes reposant sur les mathématiques, la science économique et les sciences humaines. ${ }^{48}$ Constituée par des ingénieurs et statisticiens dotés de diplômes prestigieux, la SEMA se donne pour vocation de généraliser aux mondes des entreprises et des organisations publiques des résultats de recherches appliquées dans des domaines aussi variés que l'informatique, l'urbanisme, le marketing, l'organisation logistique, etc. Conformément à cette logique de modélisation expérimentale, son rapport propose une «organisation générale du groupe Hachette » qu'Ithier de Roquemaurel décide officiellement de mettre en œuvre en juin 1969.

Pour la SEMA, la direction du groupe Hachette doit changer ses méthodes de travail afin de s'affirmer comme une direction de groupe appliquant un principe d'équivalence générale dans la gestion d'un portefeuille d'activités diversifiées. Ainsi, les conclusions du rapport se placent au-dessus des activités particulières et suggèrent à la direction générale de se détacher des tâches de gestion quotidiennes qui l'embarrassent et la détournent de la réalisation des objectifs de "politique générale du groupe ». Elles préconisent la "création d'échelons intermédiaires » et une "décentralisation » dont le " corollaire » est «l'exercice des tâches de contrôle de la direction générale ». Grâce au travail des nouveaux cadres intermédiaires, la direction générale pourra " faire faire une analyse détaillée de tous les produits ou lignes de produits ». Dans cet esprit, la politique $\mathrm{du}$ groupe est dessinée par la direction générale dans une position de surplomb parfaitement assumée et se substituant à l'ancienne implication du dirigeant principal dans la marche des affaires qui, comme l'atteste cette citation, est ouvertement critiquée :

L'amélioration de la croissance et de la rentabilité rend nécessaire une réforme des systèmes de direction et de gestion. Cette réforme (...) vise à substituer à une direction centralisée et à une gestion empirique, un système de gestion prévisionnelle budgétaire et planifiée. ${ }^{49}$

La direction n'est plus définie par un métier, celui de l'édition, mais par le modèle formel du contrôle de l'application d'une stratégie de groupe. La supervision des relations commerciales avec le monde de l'édition, activité essentielle des prédécesseurs d'Ithier de Roquemaurel, quitte le domaine d'activité du nouveau PDG, qui doit se préoccuper de l'équilibre entre toutes les activités. Suivant une préoccupation de contrôle, les consultants portent leur attention sur ce qui est le plus facilement objectivable: leurs définitions des produits correspondent $a$ priori à des unités de structure qui semblent 
aller de soi. Ainsi l'étude de la SEMA délimite formellement les groupes de départements et de filiales selon leur fonction productive : le livre, la presse, la distribution en France et à l'international. La SEMA préconise une gestion des produits à l'intérieur même des divisions ainsi instituées. Selon un point de vue normatif, le contrôle en amont de cette gestion doit permettre la croissance de la rentabilité du groupe. Ce faisant, le regard des organisateurs fait l'impasse sur l'inscription de l'entreprise dans ses nombreuses relations avec l'extérieur. Il oublie également la genèse des positions de la librairie au cours de laquelle ses propres produits et ceux issus de la coopération avec d'autres éditeurs n'ont jamais été conçus comme des objets indépendants du réseau de distribution chargé de leur distribution. L'idée même des collections comme le «Livre de poche» intègre l'anticipation de leur diffusion via les circuits de distribution de la consommation quotidienne.

À cet égard le rapport de la SEMA souligne pour le regretter que

[...] les activités de distribution sont beaucoup plus importantes que les activités

d'édition et de production.

En considérant la distribution surdimensionnée par rapport aux métiers plus créatifs de Hachette, les consultants n'ont pas conscience du travail continu d'articulation entre les services distributeurs de la librairie et les éditeurs, internes comme périphériques au groupe, à laquelle la pratique dirigeante antérieure était si attentive. Leur objectif est moins de restituer la logique des pratiques de direction antérieures, assurant quotidiennement l'équilibre des relations entre les clientèles et le monde éditorial, que de donner une visibilité à chacune des structures de l'entreprise. En isolant des pôles productifs, en considérant la distribution comme une activité distincte, les consultants espèrent donner aux actionnaires les moyens de porter un regard d'ensemble sur la consommation budgétaire et la rentabilité de chacune des activités formalisées de la librairie Hachette. Un tel cloisonnement déplace les organes de décisions de la «Librairie » (métier articulant savamment l'édition à ses circuits de vente) au " groupe » (agent gestionnaire d'un portefeuille d'activités). Ce déplacement donne, bien entendu, une justification à l'apparition d'une nouvelle catégorie de professionnels de la gestion.

\section{Les motifs politiques d'une formalisation}

Les pratiques des consultants et des nouveaux responsables fonctionnels révèlent la progression de la croyance dans les vertus d'une forme organisationnelle qui conduisent concrètement à disqualifier les anciennes pratiques de la maison. La défense d'une structure plus orientée vers la gestion des divisions que vers le service particulier intercalé entre éditeurs et consommateurs permet à de nouveaux professionnels de la gestion, diplômés et recrutés à l'extérieur, de justifier des pratiques jusque là inconnues. Ainsi, la volonté de développer des innovations dans un schéma désormais planifié, audessus des activités formalisées de l'entreprise, encourage les spécialistes du marketing à faire évoluer les contenus éditoriaux vers d'autres média que le livre alors que la librairie ne dispose pas des circuits de vente correspondants. Autre exemple, en distinguant le produit de sa mise en relation avec la clientèle, ces spécialistes mettent en cause la modalité suivant laquelle les représentants des classiques Hachette construisent leur relation de proximité avec les enseignants prescripteurs de livre, postulant ces consommateurs désireux de s'informer par eux-mêmes des innovations de contenu ${ }^{50}$.

Si les nouvelles représentations de l'organisation justifient ces pratiques professionnelles, les experts promus travaillent, en retour, à défendre cette structure en produisant des 
rapports qui cherchent à accréditer l'utilité de leur fonction. Le souci des consultants qui souhaitent "mettre en charge » les systèmes de gestion, les rendre plus opérationnels, trahit d'ailleurs une angoisse mal dissimulée sur le pari de la multiplication d'instruments qui pour l'instant n'ont que peu de prise sur le réel. Cela explique la prudence des consultants :

Dans un ensemble aussi important de réformes, l'étude trop détaillée "en chambre » du système, puis son lancement, est mauvaise car elle présente trop d'inconvénients; en fait, il faut préparer jusqu'à un certain point puis lancer et considérer que c'est la mise en place qui va constituer l'étape d'amélioration ultérieure du système..$^{51}$

Avançant avec prudence dans des activités inconnues, le langage des professionnels de l'organisation atteste d'une pensée systémique qui commande un renouvellement profond des pratiques empiriques d'exploitation. En effet, la réplique des services centraux aux niveaux des divisions est annoncée pour relayer l'effort d'organisation des véritables professionnels de la gestion. Le travail d'organiser énonce les normes d'une autorité nouvelle, puis se nourrit du travail de ceux chargés de les mettre en place à un niveau inférieur. Une forme de lien de subordination, pudiquement appelé «lien fonctionnel ", permet de détrôner les anciennes autorités de décision familiales ${ }^{52}$. Les bénéficiaires directs de cette formalisation sont les services centraux placés sous les ordres de cadres diplômés - comme par exemple « la DOTI ", Direction de l'organisation du traitement de l'information-qui revendiquent pour eux des tâches, souvent parfaitement assumées par des salariés d'implantation plus ancienne, dans un souci de bon sens gestionnaire ${ }^{53}$.

En suivant les recommandations du rapport de la SEMA, Ithier de Roquemaurel se met à la tête d'un groupe dont la représentation devient conforme aux critères d'évaluation de la sphère financière. Mais, à la suite de ce rapport, dans le même dossier, on trouve les notes de direction qui annoncent la création d'une hiérarchie de cadres dans les quatre groupes, gérant chacun une activité, à la tête desquels sont nommés des directeurs, dont l'ancien précepteur de son fils ${ }^{54}$. Cela lui permet officiellement de renouveler le comité de direction et de réduire l'autorité des directeurs de département et de ses cousins, administrateurs familiaux qui conservaient jusque là officiellement leurs responsabilités opérationnelles ${ }^{55}$. Mais, des motifs politiques les plus avouables à ceux qui le sont moins, les raisons de la mise en place de l'organisation multidivisionnelle au sein du groupe Hachette ne suffisent pas à retracer l'histoire de sa réception.

\section{Les difficultés de la transformation de l'exploitant en gestionnaire d'activités}

Dans la mise en œuvre du modèle de la SEMA, les acteurs restent confrontés aux problèmes que posent de manière récurrente les relations commerciales avec l'extérieur et les problèmes de l'exploitation des services commerciaux aux éditeurs extérieurs. Devant s'en tenir à un principe d'évaluation générale de l'activité des directeurs de groupe, le comité des directeurs de groupe ne traite plus de questions autrefois discutées par l'autorité principale de la librairie. Les réunions de direction dont nous conservons la trace montrent que les méthodes de décision deviennent, de ce point de vue, moins souples. L'évaluation des projets commerciaux relatifs au livre s'alourdit de l'intervention d'un contrôle plus strict de rentabilité et d'une politique jalouse des intérêts du groupe. Il n'est plus question pour Ithier de Roquemaurel, et son « directeur du livre », de se livrer 
aux pratiques de négociation directes qui avaient lieu au sein même du bureau de Robert Meunier. Au contraire, le nouveau dirigeant souhaite remédier à la dispersion des activités du groupe, que le fonctionnement antérieur avait suscitée.

Suivant le modèle de direction préconisé, les éditeurs périphériques se trouvent tenus à distance. À l'occasion du renouvellement des accords avec Gallimard, les interventions des responsables en "comité des directeurs de groupe» ne se concluent plus sur des décisions relatives à la prise en charge des stocks d'éditeurs ou aux auteurs, mais plutôt sur la mesure de la rentabilité du contrat, que, par ailleurs, les études de contrôle de gestion ont du mal à cerner ${ }^{56}$. En provoquant ainsi l'agacement de la famille Gallimard ${ }^{57}$, encore très imprégnée de l'esprit entrepreneurial du fondateur, Hachette manque le renouvellement du contrat de distribution avec la prestigieuse maison, qui depuis 1932 rendait possible la massification de l'offre de livres de librairie générale.

Outre la séparation de la direction générale et des exploitations propres à son métier d'origine, la mise en forme " cohérente » du groupe conduit à clore l'entreprise sur ellemême au nom d'une valorisation interne de savoir-faire mal analysés et d'une extraction des bénéfices de ses activités à son profit exclusif. C'est ainsi que, rompant avec sa tradition de coopération qui permettait de répartir les risques et de multiplier les expériences, Hachette refuse de monter un partenariat avec Bertelsmann pour créer un club de livre, que le groupe allemand lancera en coopération avec les Presses de la Cité sous le nom de France Loisirs. Estimant qu'une telle structure relève de ses seules compétences, la librairie Hachette décide de s'engager sans partenaire sur le marché des clubs du livre en méconnaissant les difficultés d'accès à une clientèle nouvelle. L'effort de trésorerie nécessaire dans ce type d'activité est regardé de manière circonspecte par les financiers qui disposent désormais des outils de contrôle d'une politique budgétaire, autrefois entre les mains $d u$ responsable principal de la maison. L'insuffisante connaissance des méthodes de ce type de commerce, liée aux timidités des soutiens bancaires, a pour conséquence le retard durable des recrutements de membres du club sur le concurrent principal, et l'échec final du club du Livre de Paris revendu en 1980 à... France-Loisirs ${ }^{58}$.

La reformulation de l'organisation de Hachette apparaît encore plus désastreuse dans les diversifications. Le groupe s'engage dans de nouvelles activités jugées indispensables à la formation d'un grand groupe de communication moderne. Mais celles-ci se révèlent très coûteuses, sans qu'aucune expérience de ces affaires ne permette à la nouvelle génération de dirigeants de réagir à temps ${ }^{59}$. L'audiovisuel, la formation, la production de disques souples sont autant de diversifications qui tournent court. En 1974, le groupe Hachette totalise 70 millions de pertes, dont une part de provisions nécessaires à la clôture des diversifications ${ }^{60}$. Ithier de Roquemaurel peut se retourner contre le directeur général recruté pendant l'été 1971, Simon Nora. Cet inspecteur des finances, ancien directeur de cabinet du Premier ministre Jacques Chaban-Delmas, est contraint de démissionner de son premier poste occupé dans le privé. Gérard Worms, jeune polytechnicien du corps des mines recruté en janvier 1972 pour épauler Simon Nora, jette alors l'éponge sur une partie de la réforme et réduit les services de planification et de marketing créés sur les conseils de la SEMA.

43 Rappelant que la librairie Hachette est à la fois un groupe gérant des participations et une structure exploitante, Gérard Worms, le directeur général simplifie la structure du groupe en revenant à des pratiques qui, sous les formalités nouvelles du professionnalisme cadre, ressemblent aux anciennes pratiques ${ }^{61}$. L'organisation inspirée 
de la SEMA n'est plus évoquée, et Gérard Worms suscite la formation de "groupes de réflexion ", où les directeurs fonctionnels centraux concernés animent la coopération interne entre différentes parties de l'entreprise, renouant avec le souci de maintenir un lien constant entre les responsables des services de distribution et les éditeurs.

L'ensemble des turbulences que traverse le groupe Hachette est perceptible dans le renouvellement brutal des formes d'archives. Si le rapport de la SEMA, les budgets produits et les notes sur les besoins de trésorerie établis par les "fonctionnels", par ailleurs intégrés aux comptes rendus des comités consultatifs d'actionnaires, sont toutes des sources qui peuvent conduire à conclure sur une modernisation de l'organisation, d'autres documents nous invitent à considérer la façon dont ces documents ont été mobilisés et leur portée réelle dans les pratiques d'entreprise. Ainsi, le moment des changements ne s'analyse pas exclusivement en suivant la lecture de ces sources normatives. La trace du positionnement d'une catégorie de professionnels : fonctionnels, directeurs de groupe, financiers extérieurs se trouve brouillée par des sources où sont révélées leurs difficultés, leur propre confrontation aux pratiques d'exploitation, voire leurs échecs. Liant leur justification à la construction même d'une structure de groupe dont l'évaluation se joue désormais autant sur des marchés financiers, extérieurs à la profession, que sur le marché des produits, le regard de ces organisateurs doit s'analyser avec prudence car il n'émerge pas des pratiques des exploitations telles qu'elles se sont historiquement construites : il répond à d'autres enjeux.

\section{Chez Panzani}

45 Si la question du renouvellement des équipes dirigeantes, chez Hachette, relevait du contexte, fortuit, d'une succession, sa démarche de diversification s'inscrivait, elle, dans un mouvement plus général qui traversa l'industrie française à la fin des années soixante et au début des années soixante-dix et qui généra une phase de concentration. Ce mouvement était une des réponses que les grands groupes, essentiellement engagés dans l'industrie lourde ou la production de biens intermédiaires, apportèrent à l'infléchissement des perspectives de croissance de leurs activités majeures, reprenant, avec quelques années de décalage, la stratégie engagée par les groupes américains qui menaçaient en se tournant vers l'Europe d'épuiser les potentialités d'investissement. Panzani Regia Scaramelli, entité issue de la fusion de 1964, fit également partie du mouvement. En 1971, elle entrait dans le giron du groupe Gervais-Danone, lui-même associé, en 1972, au groupe verrier BSN pour former le quatrième groupe du secteur industriel privé. L'entreprise sortait ainsi du petit monde de sa filière pour devenir un élément relativement marginal d'un grand groupe multiproduit. Pour gérer sa croissance celui-ci accentua sa structure multidivisionnelle et renforça ses procédures internes pour soutenir une décentralisation opérationnelle ${ }^{62}$. Tout en conservant son identité juridique, l'entreprise devint un « département » embryon d'une future «branche » qui pesa par la suite jusqu'au quart du chiffre d'affaire du groupe, et se trouva soumise à ses procédures de contrôle et d'évaluation. Cette soumission, partagée par les autres activités désormais rattachées au groupe BSN, loué pour son " dynamisme ", put apparaître comme le moyen essentiel de la diffusion de savoir-faire et de modes de gestion qui faisaient défaut à ce monde de l'entreprise familiale et des relations personnelles. À cette vision parfaitement étayée par la documentation économique et financière publiée, la mise en perspective des pratiques d'activité peut apporter sinon des nuances du moins des questionnements relatifs à la profondeur et à l'impact de ces constructions sur l'univers des pratiques. 


\section{Logiques du groupe multiproduit}

46 représentations alors à l'œuvre dans la constitution de ce groupe multidivisionnel diversifié et leurs limites. Une large part de sa cohérence présumée était tirée de l'enthousiasme pour les méthodes de gestion issues de l'univers américain, qui résultaient, pour une large part, du principe d'équivalence générale entre les activités qu'elles suggéraient. Il postulait que, s'il était possible d'aborder les secteurs de consommation de masse avec les méthodes d'encadrement du marché issues du marketing, alors la professionnalisation d'un groupe, dans ces techniques, pouvait permettre d'aborder n'importe quelle activité. Le choix de développement ne résultait en conséquence plus que des potentialités d'un marché dont l'étude devait donner les clés ${ }^{63}$. Le marché des pâtes alimentaires avait ainsi suscité l'intérêt du département études du groupe laitier qui avait préconisé un achat dans ce secteur. Au nom de ce principe général d'équivalence et par extension des pratiques commerciales développées par le groupe, le choix s'était porté sur une entreprise disposant d'une relation au marché relativement similaire à celle des activités déjà contrôlées : la distribution directe au détaillant ${ }^{64}$. La société Milliat Frères avait ainsi été identifiée et acquise en 1970. Or, le choix de l'autonomie commerciale constituait justement le fondement du déclin de cette entreprise à laquelle échappaient les marchés des grandes sociétés de distribution qui amorçaient leur explosion à la fin des années soixante. Il devint rapidement évident que les pratiques marchandes tendaient à se déplacer dans d'autres espaces ce qui conduisit Gervais-Danone à se porter également acquéreur de PRS en 1971. Cet achat prenait la forme d'un aveu de reconnaissance des limites de l'évaluation formelle de cette activité et de la méconnaissance de l'importance des réseaux et des pratiques qui les fondaient. Les limites de ce premier choix ne furent cependant pas d'abord clairement assumées, les deux sociétés étant d'abord gérées comme autonomes et complémentaires avant que les cadres de PRS ne prennent, à nouveau, la direction de l'ensemble de l'activité.

La diversification alimentaire du groupe verrier BSN relevait d'une stratégie plus constru ite que la seule diversification. Promoteur déçu d'une tentative de fusion avec SaintGobain qui lui aurait permis de constituer le premier groupe verrier mondial, BSN se tourna résolument vers l'alimentaire, à partir de 1970, pour stabiliser les débouchés de sa division de verre creux. Le monde de l'emballage voyait en effet alors s'accroitre la concurrence de nouveaux produits issus des plastiques qui permettaient d'accélérer la tendance à l'emballage perdu. Pour contrer cette menace, Antoine Riboud conçut l'opportunité d'un rapprochement contenant/contenu et poussa d'abord son groupe à prendre des positions dans les marchés des boissons puis, en 1972, dans les produits laitiers ${ }^{65}$. Il s'agissait ainsi de s'arroger un nouveau domaine de valorisation tout en faisant jouer les synergies entre deux divisions ce qui impliquait en particulier de contrôler étroitement les débouchés alimentaires pour consolider l'exploitation de la branche verrière. Cette dernière opération impliquait une planification plus poussée et donc la mise en place de méthodes de gestion jugées plus rigoureuses dans les nouvelles acquisitions. À cette fin, le groupe recourut par deux fois aux conseils du cabinet McKinsey: en 1970 pour renforcer sa structure et en 1972/1973 pour se doter d'une démarche de "planification économique et sociale " intégrant les orientations de la direction participative. La démarche dite «d'objectifs préliminaires »-OP - constituait ainsi une phase préparatoire donnant la parole aux divisions en vue d'une discussion avec 
les instances dirigeantes prenant les décisions d'allocation par le contrôle des ressources financières et des objectifs de rentabilitée ${ }^{66}$. Toutes les filiales furent ainsi amenées à construire des propositions de consolidation ou de réorientation de l'activité dans des créneaux porteurs et maîtrisables.

Les documents des OP, retrouvés dans les archives de la société, rompent clairement avec les documents de gestion élaborés antérieurement au titre de la planification ou des relations avec les actionnaires. Pour la première fois la forme et l'intention échappent à la direction et manifestent clairement sa soumission au nouvel actionnaire, à une nouvelle forme d'organisation. Jusque là en effet pas de forme figée, mais une pratique évaluant les informations nécessaires à l'intelligibilité de l'activité, des documents évoluant au gré des problèmes à résoudre et manifestant les préoccupations courantes des dirigeants comme des actionnaires. Expérience clairement nouvelle, les OP de 1973 débutent au contraire par une «note d'information » préliminaire rédigée par Jean Panzani, encore à la tête de la société :

Les réflexions et les chiffres qui constituent l'essentiel du dossier des «objectifs préliminaires" devraient se suffire à eux-mêmes, sans qu'il y ait besoin de commentaires. / La qualité de leur présentation, leur chronologie logique et leur judicieux enchaînement devraient idéalement permettre au lecteur de trouver, dans le document et de manière claire, les réponses aux questions qu'il n'aurait alors plus besoin de poser./ Malheureusement notre Société n'a pas rejoint cet état de perfection dans «la communication chiffrée et prévisionnelle», ce qui me conduit à donner quelques explications. ${ }^{67}$

49 Homme du chiffre: comptable de formation, pionnier de l'informatique de gestion, habitué des exercices de simulation, Jean Panzani, conteste ici la méthode bien plus qu'il ne déplore les faiblesses de son système de gestion. La réduction de l'entreprise à des matrices, chiffrées ou conceptuelles ne rend pas compte, selon lui d'une activité dont la compréhension requiert le recours à l'histoire de

[...] l'unité d'esprit et de fait des quinze cadres dirigeants de notre Société, image bien vivante, dynamique et motivée, d'un effort volontaire et constant de restructuration professionnelle.

De fait, en ne s'attachant qu'aux chiffres, l'exercice sur lequel doit se fonder l'évaluation de la gestion des filiales ne peut que constater l'importance des amortissements consécutifs à un effort de restructuration alors en cours et la faiblesse des résultats, souligner l'absence de perspectives dans un marché pratiquement plat et conclure en conséquence au décalage entre la recherche de relais de croissance et les caractères de l'activité principale de la société. La logique prévisionnelle conclurait sans appel à un redéploiement impératif, à la diversification ici anticipée dans les termes suivants :

La tarte à la crème de toute entreprise en mal d'expansion et de diversification, engendrant une concurrence non négligeable, la mettant face à de nombreux problèmes.

Le cadre dans lequel se fonde le nouveau groupe conduit à une critique sans appel de cette activité de toute apparence peu dynamique qui requiert des justifications différentes, le rappel d'un "esprit », d'une «motivation » pour accomplir une «tâche importante » sûrement peu susceptible d'émouvoir a priori les stratèges du groupe.

Ce type de réaction peut faire l'objet de multiples interprétations. Dans une perspective favorable à la thèse de la modernisation, elle reflète la « résistance au changement » d'un patronat condamné par l'inéluctable transformation du capitalisme français. En imposant un nouveau registre de justification susceptible de souligner les limites des pratiques anciennes, BSN aurait favorisé la «modernisation» d'un ensemble d'activités mal 
exploitées, les préparant ainsi à des normes de compétitivité et de rentabilité plus élevées. Dans une perspective critique, cette opération conjointe de dévalorisation et de valorisation de pratiques gestionnaires apparaît servir une reconnaissance de la professionnalisation par les titres et non plus par l'expérience qui sert un conflit de succession entre cadres dirigeants, la légitimité nouvelle de la professionnalisation par les titres étant alors l'apanage des jeunes générations. De fait, la grande majorité des cadres de Gervais-Danone sortit du groupe après la fusion de $1972^{68}$. Dans les deux cas, on avalise ainsi l'idée d'une transformation des pratiques dans les perspectives du modèle, la généralisation nécessaire de la forme multidivisionnelle et de ses attributs. La prise en considération des formes de pratiques antérieures à la modernisation peut permettre d'infléchir quelque peu ce point de vue tout en questionnant le processus à l'œuvre dans le changement des pratiques. Celui-ci ne peut alors plus être conçu comme la résultante d'une domination ou d'une transformation nécessaire mais comme le fruit d'une hybridation résultant des tensions à l'œuvre dans l'espace des activités.

\section{La modernisation comme hybridation}

51 La constitution d'un groupe multiproduit par acquisition d'activités nouvelles implique la mise en forme de principes d'équivalence générale susceptibles de permettre des évaluations qui sont autant de connaissances nouvelles et nécessaires. Ceux-ci peuvent résulter de principes financiers généraux et conduisent à des structures de holding qui sont susceptibles de s'appuyer sur la cooptation des dirigeants initiaux limitant par là même la diffusion de leurs compétences spécifiques. Dans le cadre de stratégies de rentabilisation par des restructurations, cette option était rendue difficile par la réduction du nombre de postes de décision qui limitait la circulation des cadres et l'incitation à la coopération. De plus, les logiques d'engagement traditionnelles se trouvaient remises en cause par la soumission à une structure de groupe tout en impliquant une sélection dont les instances dirigeantes ne maitrisaient plus nécessairement les termes. Le recours à un principe d'équivalence général, comme le fut, à partir des années soixante-dix le couple marque/innovation de produit issu du marketing, favorisa au contraire la production d'une structure de connaissance et de valorisation interne permettant d'évaluer la dynamique d'activité des divisions tout en construisant une référence commune dans laquelle devenait possible une circulation des cadres d'une activité à l'autre à défaut de parcours hiérarchiques plus traditionnels. Telle fut en gros la logique de recomposition des activités au sein du groupe BSN. Celles-ci devaient néanmoins être susceptibles de participer d'un tel modèle de fonctionnement, relever de produits et de marchés pour lesquels une telle stratégie était applicable d'où l'importance de la sélection de ces investissements.

52 À l'instar d'un grand nombre d'activités alimentaires anciennes, le secteur des pâtes alimentaires s'inscrivait difficilement dans un tel modèle. L'activité relevait d'une forme de valorisation impliquant une coopération dans l'ensemble de la filière dont la gestion supposait un engagement avec des partenaires difficiles à maîtriser et dont les résultats étaient peu capitalisables par des individualités désireuses de se construire une réputation en vue d'une carrière au sein d'un groupe. La réaction de Jean Panzani à l'impératif de mise en forme moderne de son activité comme les réactions du groupe face à cette entreprise traduisaient pour une large part ce décalage. Réciproquement, les dirigeants du groupe BSN s'interrogèrent rapidement quant à l'opportunité du maintien d'un tel type d'activité dans leur portefeuille. En conformité avec leur conception de la 
dynamique des marchés, ils ne voulurent considérer que les potentialités de développement des nouveaux produits qui nécessitaient des investissements additionnels. Pousser à son terme une telle logique consistait à rechercher une autre société si bien que PMF (Panzani Milliat Frères) fut dans un premier temps mise à l'écart. Une telle position contribua à maintenir l'homogénéité initiale de son personnel et assura la perpétuation d'une large part de ses pratiques de gestion. Le groupe dirigeant constitué au début des années soixante-dix se maintint ainsi jusqu'au début des années quatrevingt-dix. La mauvaise conjoncture de la seconde moitié des années soixante-dix lui permit paradoxalement de faire ses preuves. Confronté à une très importante dégradation des résultats de sa division verre plat, le groupe BSN dut en effet suspendre à partir de 1973, ses velléités d'investissement, d'innovation et se concentrer sur la rentabilisation des activités existantes. Dans ce cadre, PMF eut l'occasion de faire ses preuves contribuant à limiter les pertes du groupe dans les années difficiles puis, à partir de la fin des années soixante-dix, engendrant une part du résultat du groupe plus de deux fois supérieure à sa contribution au chiffre d'affaire ${ }^{69}$. Cette contribution inespérée consolida l'autonomie de la société et assura sa pérennité dans le groupe. Mieux, elle fut à la base, à partir du début des années quatre-vingts, d'une des principales divisions du groupe en passe de devenir exclusivement alimentaire et fournit, en la personne de Pierre Bonnet un des principaux collaborateurs d'Antoine Riboud. À la fin des années quatrevingt-dix, cet ancien bras droit de Jean Panzani, pouvait ainsi continuer de prêcher le respect des anciennes pratiques du secteur :

L'approche de la distribution c'est un vrai problème. Ce point de vue que dans le fond pour la marque c'est le consommateur le plus fort vient de Procter... personne ne change de boutique pour une marque. ... Il y a donc tout un problème de relations avec la distribution que j'appelle le 'marketing de proximité' qui doit être développé. On fait aujourd'hui au moins un déjeuner avec les grands patrons. Je voudrais que l'on fasse vraiment le point une fois par an. Quels ont été les drames, comment ils ont été résolus, comment on peut améliorer le global de BSN. Il faut le faire au niveau du groupe. Après on identifie les problèmes et on agit, etc... Ça peut redescendre. Il y a un apport et une considération qui font que la relation groupe/ distribution fonctionne et cela je vais le faire partout dans le monde. ${ }^{70}$

Si l'on en croit la trajectoire comme le discours de ce cadre dirigeant, la confrontation de l'entreprise avec les pratiques de groupe résulte plus d'une hybridation, particulièrement sensible dans l'actualisation de la formulation de ces pratiques anciennes, que d'une transformation radicale sous la forme d'une modernisation d'activités aux pratiques dépassables ou de la domination exclusive d'un nouveau modèle. Condamnée par les méthodes de travail censées constituer le fondement du groupe alimentaire constitué en 1973, PMF en devint un des piliers et apporta une pratique de gestion des marques d'autant plus adaptée aux caractéristiques du marché français qu'elle avait contribué à les modeler. L'attention portée aux pratiques permet ainsi de dépasser les risques d'une interprétation fondée sur un point de vue unilatéral et permet de voir les transformations organisationnelles non pas tant comme une fin que comme une étape dans un processus multidimensionnel. Le recours au modèle divisionnel mérite d'être perçu dans une configuration liant plusieurs phénomènes: une étape dans l'évolution des groupes industriels, ici le recours à la croissance externe ; les enjeux sociaux internes et externes ${ }^{71}$ propres au renouvellement du monde des cadres d'entreprises par effet de génération comme de modification des contextes d'activité par la soumission à des groupes ; et enfin des opportunités marchandes de développement de produits dont les caractéristiques, élargies à leur mode de mise en marché et donc aux pratiques qui l'instituent, influent 
largement sur leurs modes de gestion ${ }^{72}$. Dans cette perspective, l'histoire du modèle divisionnel dépasse largement le seul cadre de conditions générales d'efficacité de gestion pour susciter une réflexion sur les contextes sociaux, de pratiques et d'enjeux collectifs, qui ont pu en faire une « alternative » un moment préférée.

\section{Conclusion} haut d'activités économiques anciennes. Volontairement aveugles ou dubitatifs quant à l'originalité d'entreprises qui avaient su imprimer à ces activités la marque d'un savoirfaire essentiellement assis sur le développement de relations commerciales qui permirent d'assurer avec souplesse la diffusion en masse de leurs produits, les nouveaux groupes dirigeants échouèrent à les normaliser dans le sens promu par les analyses alors en vogue. Développées au plus près de leurs clientèles, ces pratiques pouvaient en effet difficilement être réformées unilatéralement. La ténacité avec laquelle les nouveaux dirigeants, partisans d'une modernisation économique, tentèrent de mettre en place les normes d'une organisation moderne, $\mathrm{y}$ compris dans des domaines où la genèse historique des produits leur échappait, témoigne de la profondeur de leur croyance dans le modèle qu'ils défendaient. Celle-ci s'appuyait très largement sur des outils de contrôle et de coordination semblant rendre possible la maitrise d'activités très variées et autoriser un développement harmonieux des entreprises dans une phase de croissance soutenue.

Cependant la fin poursuivie l'emporta sur les moyens mis en œuvre : pour permettre le contrôle de pratiques déjugées, le chemin le plus court fut de reformuler formellement le cadre des activités afin de le rendre conforme aux représentations sous-jacentes de l'organisation, voire à la modélisation des comportements qui le justifiait. Les organisateurs de la librairie Hachette crurent bon de favoriser un savoir-faire de producteur, indispensable à un groupe de communication moderne, au détriment d'un savoir-vendre, alors que la construction sociale de l'entreprise avait toujours su résoudre la tension entre ces deux capacités. Les objectifs prévisionnels imposés à Panzani pour dessiner une véritable stratégie de groupe dévalorisèrent les aspects quotidiens de sa tactique qui tenaient pourtant lieu de stratégie véritable.

La redéfinition des activités intégrées dans des organisations divisionnelles motivait le travail des nouveaux professionnels qui apparurent dans la conjoncture sociale de l'époque: plus jeunes, plus diplômés que leurs prédécesseurs, ils trouvaient dans le langage des structures et des produits modernisés une façon de s'affranchir de l'autorité traditionnelle en défendant une expertise fonctionnelle. Les critères d'évaluation des organisations divisionnelles légitimaient également l'usage de concepts permettant de nourrir une pensée plus générale sur la stratégie des groupes, tandis que les nouveaux pouvoirs financiers de l'entreprise ne se contentaient plus des raisonnements de marge, trop simples, des anciens cadres exploitants.

La mise en œuvre de la nouvelle organisation correspondait enfin au besoin de justifications nouvelles de toute une génération de cadres qui ne voulait plus participer à un pratique contestée du capitalisme, encore très largement imprégné de références domestiques. Leur expertise, entre autres dans le domaine du marketing, dépassant les cadres trop rigides de savoir-faire purement techniques ou purement commerciaux, se 
réclamait parfois des sciences humaines. Sans doute ce second esprit du capitalisme, analysé par Luc Boltanski et Eve Chiapello ${ }^{73}$, convint-il à une génération de professionnels en voie de faire défection aux anciennes structures entrepreneuriales. Cette génération ne put toutefois que constater la nécessaire permanence des pratiques d'exploitation qui avaient façonné le fonctionnement de leur secteur. Hachette a par exemple dû gérer la contradiction qui intervint entre une image de groupe investi dans les activités de communication et une image d'éditeur, perdue au profit quasi-exclusif de Gallimard. Le groupe BSN est devenu l'archétype du groupe moderne et innovant en termes de marketing, alors même que sa filiale Panzani pouvait continuer de se prévaloir d'un fonctionnement traditionnel. L'écart entre ces justifications nouvelles et la permanence des formes concrètes d'acti-vité trouva alors très probablement une solution à la tension qu'il engendrait lui-même en confirmant la transformation de la valorisation interne des différentes formes d'activité. La différenciation des trajectoires individuelles comme de l'ensemble des formes de gratification propres au monde économique qu'il conviendrait d'étudier plus en détail permit très certainement d'assurer la permanence du fonds de commerce initial, assise sur un personnel ancien et peu mobile, tout en motivant, par ailleurs, l'invention de nouvelles formes d'activité.

\section{NOTES}

1. Parmi l'abondante littérature sur les courants modernisateurs et leur regard sur le monde de l'entreprise, on peut se reporter à Richard Kuisel, "The American Challenge " in Seducing the French, Los Angeles, 1993, trad. fr. Le Miroir américain : 50 ans de regard français sur l'Amérique, Paris, 1996.

2. L'ouvrage emblématique de ce débat fut le très médiatique Défi américain de Jean-Jacques Servan-Schreiber, publié en 1968.

3. Ce recours fut en fait général en Europe comme le souligne Alfred Chandler, Scale and Scope. The Dynamics of Industrial Capitalism, Cambridge Mass., 1990, 615 p. ; trad. fr., Organisation et performance des entreprises, vol. 1, Les USA, 1880-1948, vol. 2, La Grande-Bretagne, 1880-1948, vol. 3, L'Allemagne, 1880-1939, Paris, 1992-1993.

4. Paradigme qui s'imposa à partir des analyses réalistes de la dynamique industrielle amorcées par Alfred Marshall et consolidées par Joseph Schumpeter et John Kenneth Galbraith que renforça le fondement historique proposé par Alfred Chandler.

5. Voir les travaux d'Alfred Chandler.

6. Après un renforcement de l'hypothèse de la stabilité potentielle des particularismes avec les débats sur le «modèle japonais ", le thème de la «mondialisation » ne rejoint-il pas ces logiques des plus classiques?

7. La proposition apparemment la plus construite, à notre connaissance, relève de Charles Sabel et Jonathan Zeitlin et des travaux qu'ils suscitèrent. Pour mémoire : Charles Sabel et Jonathan Zeitlin, «Historical Alternatives to Mass Production: Politics, Markets and Technology in Nineteenth-Century Industrialization ", Past and Present, vol. 108, 1985, p. 133-176, id. (dir.), World of Possibilities, Cambridge-Paris, 1997 ; Jonathan Zeitlin, "Productive Alternatives: Flexibility, Governance, and Strategic Choice in Industrial History ", à paraître in Franco Amatori, Geoffrey Jones (dir.), Business History Around the World at the End of the Twentieth Century, Cambridge. 
8. Olivier Zunz, L'Amérique en col blanc, Paris, 1991.

9. Robert Freeland, "The Myth of the M-Form? Governance, Consent and Organizational Change ", American Journal of Sociology, 102, 1996, p. 483-526.

10. Charles Sabel et Jonathan Zeitlin, op. cit. ; le travail récent de Robert Boyer permet également de suivre cette évolution, «Evolution des modèles productifs et hybridation. Géographie, histoire et théorie », Document du CEPREMAP, n 9804, décembre 1997.

11. Aucune institution n'est par exemple comparable au Hagley Museum.

12. Les choix de nationalisation se portèrent, de fait, sur les entreprises les plus susceptibles d'être précocement organisées selon les modèles de la grande organisation à l'activité diversifiée ou complexe.

13. On dénomme ainsi une entreprise qui changea plusieurs fois de raison sociale au cours de la période évoquée : Établissements Panzani durant les années cinquante, Panzani La Lune de 1959 à 1964 avec une structure de holding, Panzani Regia Scaramelli de 1964 à 1971 puis Panzani Milliat Frères. Le recours à la dénomination en Société Panzani vaut essentiellement en raison de la construction de cette entité autour de la marque correspondante comme de la permanence de l'équipe dirigeante sur cette période.

14. Cet accès résulte de travaux menés par ailleurs dans le cadre de doctorats. Les archives de la librairie Hachette ont fait l'objet d'un versement auprès d'une institution privée, l'IMEC. Celles de la société Panzani ont été consultées directement dans le cadre de cette société.

15. Les guides touristiques de la librairie Hachette témoignent de l'intégration du mode de distribution dans la conception même du produit. En effet, leur structure interne, suit les étapes du parcours du voyageur sur les nouvelles lignes de chemin de fer. D. Nordmann, «Les guidesJoanne, ancêtres des guides bleus ", in P. Nora (dir.), Les Lieux de mémoire, La Nation, vol. 1, Paris, 1997, p. 1055.

16. À ce propos, voir A.-M. Thiesse, Le Roman du quotidien, lecteurs et lectures populaires à la Belle Époque, Paris, 2000, p. 19-27.

17. La librairie Hachette assure pendant la guerre la distribution de nombreux journaux qui éditent des romans populaires. Le directeur commercial du Matin, Guy Schoeller est recruté chez Hachette au lendemain du conflit pour succéder au directeur des messageries Eugène Delessalle.

18. G. Suchet-Duchaux, ronéotypé, Histoire de la société Hachette. Cet ancien chartiste de formation a conçu le projet d'une histoire de l'entreprise où il a passé une carrière d'illustrateur au département des «Classiques».

19. Dirigeant principal de l'entreprise devenue société anonyme en 1919 , le président du conseil d'administration, Edmond Fouret, se sent sans doute plus puissant dans son métier que son coactionnaire la Banque de Paris et des Pays Bas, dont il est membre du conseil d'administration. En 1926, il est encore en mesure d'imposer son point de vue dans le pacte d'actionnaire qui le lie avec la banque. Soucieux de préserver l'autonomie et l'originalité de l'activité de la librairie qui fonde la légitimité de la famille fondatrice, il déclare : la Librairie n'est ni un commerce, ni une industrie, mais bien les deux à la fois, archives Paribas, 570-573. Cette conception qui efface la frontière classique séparant les marchands des producteurs reste la logique dominante des pratiques dirigeantes sous sa présidence jusqu'en 1952, et sous celle de son successeur, Robert Meunier du Houssoy, jusqu'en 1967.

20. Aucune synthèse régulière sur les ventes n'est apparue dans les archives. Leur volume apparaît de temps à autre dans les rapports d'activité. Mais cette entreprise est très soucieuse de ne pas communiquer trop de chiffres sur son activité d'intermédiaire. Jusqu'en 1964, elle ne transmet aux actionnaires qu'un produit brut d'exploitation, qui ne peut être pris que comme une approximation de la croissance de volumes d'affaires sur des produits extrêmement variés. Significativement l'activité de distributeur de presse et de distributeur de livre est confondue dans ces chiffres. Le produit brut d'exploitation de la librairie passe de $5237054882 \mathrm{~F}$ en 1952 à $260836603 \mathrm{NF}$ en 1965 soit un triplement en francs constants. Pour avoir une idée plus précise 
sur la progression des volumes d'un produit très caractéristique du métier de Hachette, le « Livre de poche » qui est lancé en 1953 est vendu à 14000000 d'exemplaires en 1960, 17000000 en 1961, et 22000000 en 1963. Aucune maison d'édition ne donne de chiffres à ces dates, parce qu'aucune d'entre elle n'est tenue, de par ses statuts, d'informer son public sur ses ventes.

21. Ces réunions semblent au cœur de l'activité de commandement de Robert Meunier du Houssoy: les autres dossiers contenus dans ses archives conservent le caractère de dossiers privés, confidentiels, non discutés au delà de son propre bureau, ou de ses correspondances privées. Ce n'est qu'en 1967, qu'un compte-rendu plus formel, mentionne explicitement la « reprise » d'un comité de direction. Depuis la disparition des administrateurs de la génération de Meunier qui se rencontraient tous ensemble, sans doute plus régulièrement. Robert Meunier n'avait donc pas cru bon de réinstaurer une réunion de direction générale autre que celle-ci.

22. Avec ces remplacements, la nature de ces réunions ne change pas ni sur la forme ni sur le fonds. Toujours les mêmes questions y sont traitées. A partir de 1964, la succession de Robert Meunier semble s'annoncer : Ithier de Roquemaurel est souvent présent à côté de son oncle, et le remplace de plus en plus souvent.

23. Archives IMEC, fonds Hachette, S3C19B6, et S3C5B1.

24. Le compte rendu de ces réunions hebdomadaires est rédigé par Henri Gautrelet, le bras droit de Robert Meunier. Ces comptes rendus prennent place parmi l'ensemble de ses lettres et notes réunies dans son correspondancier. Ils suggèrent ainsi qu'ils pouvaient être utilisés comme un aide mémoire relatif aux décisions prises. Dans les années 1960, le successeur d'Henri Filipacchi, Guy Schoeller se montre de plus en plus attentif à la façon dont ces notes sont rédigées, ce qui accentue leur caractère de procès verbal fixant une décision ou une déclaration.

25. D'autres dossiers de Robert Meunier pourraient être évoqués. Mais ils ont toujours un caractère plus personnel, où l'exercice de la direction n'apparaît guère publiquement, ce qui renvoie le mode d'autorité dont il use dans le registre du privé et rappelle l'autorité « naturelle » $\mathrm{du}$ propriétaire. Ainsi les relations importantes avec les actionnaires descendants de Louis Hachette, la prise en charge des rares revendications de salaire, et certaines relations avec des personnes d'influence comme Pierre Lazareff, directeur de France Soir, à ce titre, entrepreneur de presse du groupe Hachette, sont-elles toutes entre les mains du président de la librairie qui les gère de façon discrétionnaire. Outre ces dossiers, Robert Meunier conserve la main sur des registres comptables faisant le point sur l'état des collections de la librairie dont il a sans doute assumé la charge dès son apprentissage à la librairie, qui remonte au début du siècle.

26. Comptes rendus d'une série de réunions de 1948, archives de l'IMEC, fonds Hachette, S3C19B6 et S3C5B1

27. Témoignage de Jacques Chassepot, le 23 janvier 1996. Cet administrateur civil du ministère de l'Économie et des Finances est recruté en 1964 comme attaché financier.

28. Dans un entretien avec Maurice Dumoncel, qui dirige alors la maison d'édition Tallandier, orientée vers les produits de littérature "populaire ", la dimension affective et interpersonnelle qui lie ceux qui se considèrent comme des «chefs de maison» apparaît nettement: Personnellement je me suis d'ailleurs toujours entendu avec Meunier du Houssoy. Il a sûrement facilité ma promotion à l'intérieur et à l'extérieur de la librairie. Il est frappant de noter que cet éditeur "périphérique » apprécie une carrière qui l'a toujours conduit entre sa propre maison et la librairie Hachette, où, il est vrai, il a assumé un moment les fonctions de directeur général de la filiale de Hachette, Fayard. Regrettant ses déboires avec les dirigeants ultérieurs, M. Dumoncel s'exclame : L'idée d'un procès avec Tallandier était insupportable à Meunier du Houssoy, entretien avec Maurice Dumoncel, le 26 janvier 1996.

29. Les témoignages sur les relations de Gaston Gallimard avec les responsables de la maison de distribution insistent sur une atmosphère d'égalité qui régnait lors des premières relations commerciales entre les responsables des maisons. Ainsi Édouard de Cossé-Brissac, membre par alliance de la famille Hachette, compare-t-il les deux époques : C'était des discussions entre patrons 
et personne d'autre. J'ai eu des récits de l'accord Gallimard. Ça se passait au Sphynx qui était le bordel chic d'avant-guerre et c'est là que se sont discutés les principaux articles de la collaboration entre Gallimard et Hachette.

30. J.-C. Édeline, La Diffusion du livre par Hachette en France, 1942. On ne dispose pas d'information sur cet auteur. Sa brochure qui ne comporte aucun lieu de publication ni de mention d'éditeur, est cependant très documentée, ce qui permet de penser qu'il a eu un accès très privilégié aux messageries Hachette du livre, alors dirigées par Henri Filipacchi

31. Cette distinction réalisée en interne par les services commerciaux permet aux éditeurs de retrouver leurs comptes, sans se confondre avec les éditeurs de littérature populaire. C'est pourtant les mêmes canaux de distribution qui font de leurs rééditions des succès " populaires ».

32. La question de l'hypothèse de clôture est au fondement de l'analyse économique de la firme. Pour une présentation classique voir Edith Tilton Penrose, Facteurs, conditions et mécanismes de la croissance de l'entreprise, trad. fr., Neuilly, 1963.

33. L'essentiel des créations d'activités eut lieu dans l'entre-deux-guerres conduisant à une situation de surcapacité de production dès la veille de la Seconde Guerre mondiale qui donna lieu à un système de contingentements jusqu'à la fin des années cinquante.

34. Ces éléments ont bien évidemment joué mais sur un mode mineur. Par rapport à la suite de notre propos, il convient de noter qu'une explication fut construite ex post: celle de «l'idée de génie » d'un conditionnement, la cellophane, " adapté aux contraintes » des nouvelles formes de distribution liées au renouvellement du commerce de détail avec le développement du libreservice à partir des années cinquante. Il ne s'agit rien de moins que d'une mythologie, encore véhiculée parmi les études de cas de l'enseignement du marketing, qui contribue à produire des arguments en faveur de cette discipline. Le choix eut bien lieu, avec des conséquences, mais dut être, difficilement, imposé. Ce type d'explication, courant à partir des années soixante-dix, correspond en fait à la transformation des représentations des activités concourant à la diffusion en masse de produits et à leur valorisation au sein des activités économiques.

35. En 1959 avec la division pâtes alimentaires de Biscuits Brun Pâtes La Lune et, en 1964, avec Regia Scaramelli.

36. Les innovations en matière de gestion reposèrent sur l'intégration de professionnels issus d'entreprises concurrentes et sélectionnés lors des fusions menées avec ces dernières dans le courant des années soixante. Les nouvelles méthodes et fonctions, caractéristiques de l'évolution des entreprises françaises durant cette décennie, telles la planification, la gestion des achats puis le marketing et les ressources humaines, furent ainsi implantées dans l'entreprise.

37. Entretien avec Jean Panzani, septembre 1996.

38. Courrier de Jean Panzani à Charles Guichard, 26 février 1966.

39. Ibid.

40. Cette configuration est à la base des analyses que les économistes donnent aujourd'hui des relations de confiance. Elle peut, à défaut, être résolue par la formation d'une organisation.

41. Charles Guichard soumet ainsi la tarification de la margarine Astra pour les livraisons. Cet échange permet de rappeler les caractéristiques matérielles et économiques (encadrement des prix) du produit. Courriers de décembre 1965.

42. Ces développements peuvent être extrêmement divers, depuis l'adaptation des livraisons à l'organisation interne au client, qui fait l'objet d'une rémunération assise sur la définition de la prise en charge des fonctions mises en œuvre, jusqu'à l'évocation de nouveaux produits susceptibles d'élargir le volume d'affaire.

43. L'appui sur le commerce de détail traditionnel, qui constituait une stratégie commerciale plus classique par la constitution de vastes services commerciaux internes, supposait au contraire soit de lourds investissements en personnels ou en marges pour assurer une diffusion dans les centaines de milliers de points de vente présents dans les années cinquante et soixante, soit un cantonnement aux marchés les plus aisément défrichables ce qui limitait la diffusion des 
produits à la région parisienne et aux autres grands centres urbains par conséquents lieux de la plus vive concurrence. Cette approche avait la faveur des industriels soucieux de la maîtrise de leur commercialisation, comme les sociétés distribuant des produits spécifiques ou celles issues des multinationales étrangères qui disposaient d'importants moyens financiers ou en marges. En s'appuyant sur la distribution organisée des sociétés à succursales multiples, des coopérateurs ou encore des magasins populaires, Jean Panzani faisait le choix de la négociation mais pénétrait également le marché des agglomérations rurales encore relativement mal desservi.

44. Il perdit la propriété de son entreprise dès la fin des années cinquante, pour partie en raison de difficultés de financement de ses politiques commerciales.

45. Proportionnellement, le service commercial apparait ainsi nettement plus instable que l'emploi ouvrier.

46. Une très large part y fit l'intégralité de sa carrière formant une génération qui ne quitta la société que dans le courant des années quatre-vingt-dix.

47. L'analyse de la chronologie de ce type de configuration et son impact sur les structures industrielles dépasse, de loin, le cadre du présent article. Pour une présentation des perspectives ouvertes par ce type de point de vue, voir Jonathan Zeitlin, "Productive Alternatives », op. cit.. Notons cependant que les idéaux d'encadrement du marché par des techniques marketing permettant une stabilisation des flux favorable aux modèles de rationalisation supposent soit des capacités de vente directe soit un relatif émiettement de la structure de distribution. Le «cas français » apparaît à cet égard très différent du cas américain où, dans l'alimentaire par exemple, le rapport de tailles relatives est demeuré relativement favorable au monde industriel jusque dans le courant des années quatre-vingt-dix. On peut voir là une des significations possibles de la notion « d'étroitesse du marché » qui joue un rôle important dans les dynamiques industrielles.

48. Henri Deroy, brochure diffusée après le décès de cet ancien administrateur banquier de la librairie Hachette, vice-président, puis président de la Banque de Paris et des Pays-Bas, 1955-1966.

49. Rapport de la SEMA, juin 1969, archives IMEC, fonds Hachette, S3C22B4.

50. D. Bourdeaux et Y. Brinai, Regards croisés, Hachette, 1995. Ce petit ouvrage, diffusé en interne, et publié à l'occasion du départ à la retraite de deux cadres maisons, donne un témoignage ironique sur la prise de pouvoir de diplômés de HEC à la fin des années 1960 et leur redéfinition des méthodes de vente. Leur échec le plus frappant est la fabrication d'un catalogue pédagogique très lourd. Un dispositif de fascicules à intercaler dans le catalogue est alors conçu à l'usage des enseignants qui, suivant ce système, doivent s'informer des innovations pédagogiques.

51. Rapport de la SEMA, juin 1969.

52. C'est ainsi qu'est nommée la relation entre les responsables de fonctions localement assumées et les nouveaux responsables de ces fonctions au niveau central de la direction générale. Organisation générale du groupe Hachette, mars 1970, document communiqué par un des anciens directeurs de groupe. Ce document est produit par M. Teper, ingénieur centralien, professeur de management, recruté par Ithier de Roquemaurel qui crée à cette occasion une Délégation générale au plan et aux méthodes. Lors d'une des réunions où il est présenté, les divers responsables éditoriaux s'inquiètent de ses attributions sur le papier.

53. C'est le cas du chef des services achats de l'économat qui, indigné de voir trois rapports de «fonctionnels » sur sa propre activité, produits derrière son dos, communique à ses supérieurs une note fouillée sur un service qu'il gère depuis très longtemps, et dont il souhaite démontrer qu'il en connaît parfaitement le fonctionnement. Ces rapports sont mentionnés dans une note de ce chef de service au directeur des services d'administration générale du 19 novembre 1970, archives IMEC, fonds Hachette, S6C57B1.

54. Ithier de Roquemaurel dispose d'un "avant-projet " ponctuellement corrigé au stylo et datant du 27 Juin 1969 où toute l'organisation de la librairie Hachette est reformulée. Il prend le soin d'y énoncer les titres, mais aussi les diplômes et les âges de ses nouveaux directeurs de 
groupe, présentés sous leurs atours de jeunes professionnels. L'agrégation de lettres, le diplôme de l'institut d'études politiques, côtoient les titres des « fonctionnels » issus de l'ENA et de l'École centrale, rapport de la SEMA, source citée.

55. Archives IMEC, fonds Hachette, S3C22B4. Les réactions des cousins d'Ithier de Roquemaurel ne se sont pas faites attendre, furieux d'apprendre par Le Monde la modification des structures intervenues. Lettres de Claude Labouret et de Didier Fouret du 19, 20 juillet 1969 et 5 août 1969.

56. Un compte rendu du «comité restreint » du 17 juillet 1970 formé avec les administrateurs familiaux écartés montre que les études du contrôleur de gestion sont alors difficiles à utiliser pour prendre une décision. Ithier de Roquemaurel affirme que l'avis de son directeur de groupe reste le meilleur. C. Labouret insiste pour que soit mesurée le coût des services de distribution particuliers demandés par Gallimard. Cette attitude m'a été confirmée lors d'un entretien avec $C$. Labouret. Ce responsable "né dans l'édition » affirme avoir apprécié le travail de ce contrôleur de gestion qui avait à s'ajuster au plus près des pratiques de distribution pour que soit proposée une réponse argumentée à Gallimard.

57. Dès avril 1965, des comptes rendus de réunions chez Meunier montrent que Gaston Gallimard s'offusque des hésitations d'Ithier de Roquemaurel, directeur général adjoint, neveu et dauphin de Robert Meunier, à le rencontrer en tête à tête. Ithier de Roquemaurel souhaite imposer à ces réunions ses propres intermédiaires pour tenter de régler les conflits quotidiens opposant Hachette à sa maison. Ainsi repère-t-on, dans la forme connue des anciens comptes rendus de réunion, un détail de la négociation continue avec Gallimard, qui révèle une lésion, symbolique mais certainement profonde, dans la relation de confiance qui unissait jusqu'à présent les deux maisons: NRF: M. Gaston Gallimard ayant écrit qu'une visite de Monsieur de Roquemaurel accompagné par Monsieur Schoeller et Monsieur de Fallois "n'avait pas de sens", il est convenu que Monsieur de Roquemaurel annulera le rendez-vous prévu ce même jour. Compte rendu de réunion du 21 avril 1965, archives IMEC, fonds Hachette, S3C5B1.

58. Dans le circuit de distribution particulier des clubs de livres, l'entreprise investit principalement dans les efforts de recrutement de clients membres qui s'engagent, moyennant un abonnement, à acheter une certaine quantité de livres publiés par le club du Livre de Paris. Cela suppose des campagnes coûteuses pour recruter les consommateurs potentiels, et des avances de trésoreries considérables pour attendre le paiement du client dont le comportement d'achat peut d'ailleurs être insuffisant. Dans ce métier dont la librairie Hachette ignore tout, ce recrutement est confié à une filiale de courtage. En juin 1973 une note conjointe de la direction des affaires industrielles de Paribas et des contrôleurs de la librairie Hachette, relève qu'en 1972 cette activité a perdu 3 MF pour 7 MF de chiffre d'affaires, et que les besoins en trésorerie sont très importants, archives Paribas 570-573. Gérard Worms affirme dans une note de synthèse confidentielle, datée du 6 mai 1976, communiquée au président qui vient d'être installé par Paribas, que les méthodes de recrutement par courtage ont dû être changées au profit des techniques marketing de vente par correspondance : "mailing, parrainage, annonces presse ", archives IMEC, fonds Hachette. S3C29B2.

59. Dans un entretien Gérard Worms, recruté comme directeur général adjoint en janvier 1972, admet l'inexpérience des dirigeants recrutés, en même temps que lui, dans le service public. Ces hauts fonctionnaires d'origine assumèrent toutes ces diversifications, avant de devoir solder les comptes.

60. Rapport d'exercice, 1974.

61. Note de synthèse de Gérard Worms du 20 février 1975, archives IMEC, fonds Hachette, S3C29B2.

62. La structure multidivisionnelle de BSN à partir des années 1970 fut pratiquement un cas d'école, du moins dans sa présentation. Cependant, elle résultait plus, aux dires de son président, Antoine Riboud, d'un renforcement de la coordination par le haut entre les divisions par la 
création d'un «état-major» que d'une décentralisation des décisions opérationnelles. Voir «Entretien avec Antoine Riboud », Entreprise, 787, 10 octobre 1970.

63. Cette conception est par exemple parfaitement explicite dans la relation de la «philosophie » du groupe Gervais-Danone proposée par L'Expansion en 1968: Si les dirigeants vendent bien des produits laitiers, ils pourraient tout aussi bien, en utilisant leurs techniques et en les adaptant, réussir sur d'autres marchés comme ceux des roulements à billes ou des produits pharmaceutiques. On notera bien entendu la place accordée aux «dirigeants » et le rôle de ce type d'affirmation pour consolider l'entreprise de dévalorisation de l'ensemble des savoirs de métiers.

64. Alors à la fois stratégique car permettant une véritable application des méthodes marketing et nécessaire à la distribution de produits frais.

65. Entretien cité par Pierre Labasse, "De BSN à Danone", in Jacques Marseille (éd.), Les Industries alimentaires, Paris, 1997.

66. Pour une présentation plus détaillée de cette procédure et le rappel du contexte de développement de la planification dans les années soixante-dix, voir Raymond-Alain Thiétart et Luc Marmonier, "Les nouveaux problèmes de structure et de gestion des entreprises ", in Maurice Levy-Leboyer (dir.), Histoire de l'industrie française, Paris, 1996, p. 468-497.

67. Cette citation ainsi que les suivantes sont extraites des Objectifs préliminaires 1974-1978, du 5 avril 1973.

68. Ces points ont fait l'objet, dès l'époque, de nombreuses mises au point. Sur l'usage du modernisme au sein du monde patronal voir Pierre Bourdieu et Monique Saint-Martin, «Le patronat ", Actes de la recherche en sciences sociales, $n^{\circ} 20-21,1973$, p. 14-34; sur la dynamique du groupe des cadres durant les années soixante voir Luc Boltanski, Les Cadres, Minuit, 1981 ainsi que Luc Boltanski et Ève Chiapello, Le Nouvel esprit du capitalisme, Paris, 1999 ; sur le cas particulier du groupe BSN voir Michel Bauer et Elie Cohen, Qui gouverne les groupes industriels ?, Paris, 1981 et les rapports d'études du CORDES, sous la direction de Lucien Karpik, dont cet ouvrage est issu.

69. En 1973, PMF représentait $5.3 \%$ du CA groupe et $7 \%$ du résultat net, pour une part du CA sensiblement identique sa part du RN se montait à $37 \%$ en 1976 puis demeura supérieure à $10 \%$, rapports d'activité $\mathrm{BSN}$.

70. Entretien avec Pierre Bonnet, juin 1998.

71. En particulier les formes de justification qui fondent l'adhésion à une forme d'activité au sens établi par Luc Boltanski et Eve Chiapello, op. cit.

72. Pour une approche de cette question voir Robert Salais et Samuel Storper, Les Mondes de production, Paris, 1994.

73. L. Boltanski et E. Chiapello, op. cit. 